\title{
Regulation of Indoleamine-3,5-dioxygenase 1 (ID01) in Lung Epithelial Cells by Coronaviruses (SARS- CoV) and Cytokine Signaling
}

Ramana Chilakamarti ( $\sim$ ramana181@gmail.com )

https://orcid.org/0000-0002-5153-8252

\section{Research Article}

Keywords: ID01, Interferon, SARS-CoV-2, Tryptophan metabolism, Cytokines, Lung, Stat1

Posted Date: September 7th, 2021

DOl: https://doi.org/10.21203/rs.3.rs-871230/v1

License: (c) (1) This work is licensed under a Creative Commons Attribution 4.0 International License.

Read Full License 
Regulation of Indoleamine-3,5-dioxygenase 1 (IDO1) in Lung Epithelial Cells by Coronaviruses (SARS-CoV) and Cytokine Signaling

Chilakamarti V. Ramana

ORCID ID: /0000-0002-5153-8252

* Corresponding author

E-mail address: ramana181@gmail.com (C.V.Ramana). 


\section{Abstract}

Interferons (IFNs) and proinflammatory cytokines play an important role in the innate immune response to respiratory viruses, including coronaviruses (SARS-CoV). Metabolic profiling in the serum samples of coronavirus disease-19 (COVID-19) patients revealed altered cholesterol and tryptophan metabolism. Indoleamine-3,5-dioxygenase (IDO1) is the key enzyme involved in the tryptophan catabolism and induced by interferons and inflammatory cytokines. The regulation of IDO1 in immune cells and cancer was extensively studied. In this study, IDO1 regulation in human lung epithelial cells by coronaviruses and respiratory viruses as well as inflammatory cytokines was investigated. SARS-CoV-2 was a potent inducer of IFN-regulated metabolic enzymes such as IDO1, Cholesterol-25-hydroxylase (CH25H), Spermidine acetyltransferase (SAT1), and Sterile alpha motif and histidine/aspartic acid domaincontaining protein (SAMHD1) at RNA levels in Calu-3 cells. Reconstitution of A549 lung epithelial cells with Angiotensin-converting enzyme 2 (ACE2) was necessary and sufficient to induce IDO1 at RNA levels. Influenza A virus (IAV) suppressed IDO1 RNA levels in a non-structural protein (NS1)dependent manner in NHBE cells. In contrast, IDO1 RNA levels were dramatically induced in the lungs of mice infected with a reconstructed 1918 H1N1 influenza virus. Treatment of A549 cells with either type I or type II interferon induced IDO1 RNA levels. Furthermore, IDO1 levels were significantly higher in the lung tissues of COVID-19 patients in comparison with healthy controls. A mix of proinflammatory cytokines dramatically induced IDO1 and chemokine RNA levels in lung epithelial cells in a cell culture model, simulating the gene expression pattern in the lung tissue samples of COVID19 patients. Furthermore, hypertonic saline solution (HTS) dramatically abrogated the gene expression induced by cytokine mix in human lung cells. The IDO1 protein interaction network included transcription factors STAT1 and STAT3. These studies suggest that IDO1 inhibition may be a potential therapeutic target in the treatment of viral and inflammatory diseases. 


\section{Introduction}

SARS-CoV-2 infection and severe COVID-19 is the leading cause of death in the intensive care units (ICU) of the critical care hospitals around the world $(1,2)$. A variety of high-throughput studies in the last two years have provided novel insights in the area of coronavirus-host interactions including cytokine mediated signal transduction pathways, gene expression, and identification of potential drug targets (3-8). Gene expression profiling by microarrays and RNA-seq enabled the global monitoring of cell-, tissue-, and disease-specific gene expression patterns in the human lung cells in response to SARS-CoV-2 and in COVID-19 patients $(3,4,7,8)$. These methods provide simultaneous quantitative analysis of differential expression levels of thousands of genes on a single platform. Multiplex antibody assays provided important information on the temporal regulation of cytokine levels, including type I and type II

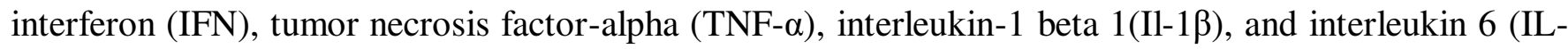
6) in the bronchoalveolar lavage fluid (BALF) of COVID-19 patients (9). Multilevel proteomics revealed the function of SARS-CoV-2 encoded viral proteins and their interactions with the host genome and global alteration of signaling pathways such as Transforming growth factor-beta (TGF- $\beta$ ) and autophagy in A549 human lung cells reconstituted with viral entry receptor ACE2 (10). Furthermore, metabolic studies revealed high levels of tryptophan catabolic product kynurenine in the serum of COVID-19 patients $(11,12)$. Tryptophan is an essential amino acid for cell survival and the intracellular enzyme that breaks down tryptophan to kynurenine is known as Indoleamine-3,5-dioxygenase or IDO1(13). Constitutive low expression of IDO1 was reported in multiple tissues including peripheral blood and enhanced expression in several cancer cells (14). The role of IDO1 in the context of immune responses and tumor cells was well established $(13,14)$. Tumor cells up-regulate IDO1 to suppress T cell activity by two mechanisms-directly starving $\mathrm{T}$ cells of essential tryptophan and triggering $\mathrm{T}$ regulatory (Treg) cell development to suppress immune responses. Inhibition of IDO1 can reduce the number of 
regulatory T cells and restore T-cell function (15). Proinflammatory cytokines such as TNF- $\alpha$, IL-1, IL6, and interferon (IFN) treatment induced IDO1 RNA and enzyme levels (16-19). A healthy microbiome in the human gut and tryptophan metabolism is required for neurotransmitter serotonin and neuropeptide melatonin synthesis. A diet low in tryptophan for 8 weeks in aged mice altered the gut microbiota composition, promoted inflammatory cytokine synthesis resulting in systemic inflammation (20). IDO1 was induced in multiple cell types in response to type I (IFN- $\alpha / \beta)$ and type II interferon (IFN- $\gamma$ ). However, IFN- $\gamma$ was more potent than IFN- $\alpha / \beta$ in the induction of IDO1 in most cell types (Supplementary data). Influenza infection of respiratory epithelial cells induced IDO1 expression simultaneously with IFN- $\lambda$ expression (21). Direct treatment of cells with IFN- $\lambda$ up-regulated IDO1 activity. Furthermore, IFN- $\lambda$ knockdown by Si-RNA treatment inhibited IDO activity during influenza infection in the lung epithelial cells (21). The interferon-inducible IDO1 was demonstrated as an antimicrobial effector mechanism that limits pathogen proliferation (22). The products of tryptophan catabolism by IDO1 such as 3-hydroxykynurenine and reactive oxygen species (ROS) produced by the action of proinflammatory cytokines promoted apoptosis in human retinal epithelial cells (17). Products of the kynurenine pathway stimulated by interferon treatment including quinolinic acid and picolinic acid were reported to have antiviral properties $(23,24)$. Modulating the cell metabolism to their advantage is a key aspect of virus-host interactions. Transcriptional induction of the host catabolic enzymes by interferon depletes the essential components of cell survival such as the amino acid, lipid, nucleotide, and polyamine reserves to limit pathogen proliferation. Previous studies have shown the critical role of type I interferon signaling and transcription factors in response to SARS-CoV-2 infection and in COVID-19 patients $(25,26)$. In this study, regulation of IDO1in human lung epithelial cells in response to respiratory viruses, interferon, and proinflammatory cytokines was investigated. 


\section{Materials and Methods}

\subsection{Gene expression datasets and Bioinformatics}

Human tissue gene expression data of IDO1 was retrieved from the GTEx database. Lung cell atlas data were downloaded from Human Protein Atlas. Microarray data in GEO datasets (GSE147507) (GSE47960) (GSE156295), (GSE70445), and GEO profiles were retrieved from the Pubmed (NIH) resources. Geo datasets were analyzed with the GeoR2R method (NCBI). Gene expression in human lung cell lines infected with respiratory viruses and COVID-19 patients was retrieved from Immgen RNA seq SKYLINE resources. Additional gene expression datasets were downloaded from the H2V database, Immgen browsers, Coronascape, and Signaling Pathway Project. Cluster analysis was performed using gene expression software tools (27). Protein-protein interactions were visualized in the STRING database (28). Gene ontology (GO) and signaling pathway analysis was done in Metascape (29). Interferon-related data was retrieved from WWW.Interferome.org. Gene-specific information was retrieved from standard bioinformatics websites.

\section{Results and Discussion}

\subsection{Profiling cell-specific expression of IDO1 in the human lung}

IDO1 was expressed at low levels in several tissues and up-regulated in many cancer cells $(13,14)$. Tissue distribution analysis in the GTEx database of human tissues revealed that IDO1 was highly expressed in the lung. colon and esophagus mucosa (Figure 1). These organs are in direct contact with respiratory or food-borne pathogens. IDO1 is predominantly localized in the cytoplasm. The other intracellular locations of IDO1 includes the nucleoplasm, vesicles and mitochondria. The human lung is a complex organ, composed of more than 30 cell types including type I (AT1) and type II (AT2) epithelial cells, club cells, ciliated cells, endothelial cells, and immune cells. The Human Protein Atlas (HPA) database contains detailed information on tissue and cell RNA expression levels of genes. Cell-specific 
RNA and quantitative expression analysis in the HPA human lung cell atlas revealed that IDO1 was highly expressed in the macrophage c-2, club c-7, and endothelial c-9 cell population. AT2 cell expression levels of IDO1 in c-1 and c-6 were very low and undetectable, respectively (Figure 2). The localization of IDO1 in club cells (also known as Clara cells) may be of functional importance as they are involved in the protection of airway tissue from the toxic chemicals that reach the lung via inhalation. Club cells are essential for the integrity and regeneration of airway epithelium and express several enzymes involved in drug and xenobiotic metabolism such as cytochrome P450, monooxygenases and glutathione peroxidases (30). IDO2 catalyzes the same reaction as IDO1 but with much less efficiency than IDO1 and approximately 50\% of caucasians have genetic polymorphisms that abolish its expression (31). IDO2 expression was detectable in mac c-2 and T-cell c-3 cell population. Furthermore, Secretoglobin (SCGB1A1) representing a cell-specific marker was highly expressed in club cells (Figure 2). Heat map representation of gene expression profile of IDO1 along with AT2, AT1, club, ciliate cellspecific markers were shown for a comparison purpose (Figure 3).

\subsection{Regulation of IDO1 expression by coronaviruses}

Most of the gene expression studies on SARS-CoV-2 were carried out in well-established human lung cell lines such as Calu-3, A549, and NHBE. Basal expression of IDO1 was very low in these cell lines. Infection of Calu-3 cells with SARS-CoV-2 dramatically induced IDO1 RNA levels within 12 hours and further enhanced by 24 hours (Figure 4A). Mock-infection for 12 or 24 hours served as controls. In contrast, SARS-CoV-1 induction of IDO1 RNA levels was much lower at 12 and 24 hours in Calu-3 cells. Interferon-stimulated genes (ISG) play a major role in antiviral response and metabolic reprogramming of the cells and are essential to limit the pathogen spread (32). Some of the well-known metabolic effectors of interferon signaling including Cholesterol-25-hydroxylase $(\mathrm{CH} 25 \mathrm{H})$ involved in cholesterol metabolism, Spermidine/spermine acetyltransferase 1 (SAT1) involved in polyamine 
regulation, Sterile alpha motif and histidine/aspartic acid domain-containing protein 1 (SAMHD1) involved in innate immunity and IDO1 involved in tryptophan metabolism were induced by SARS-CoV2 in Calu-3 cells within 24 hours (Figure 4B). CH25H represses the cholesterol synthesis by converting cholesterol to 7-hydroxy cholesterol. SAT1 and IDO1 are rate-limiting enzymes in the catabolism of polyamines and tryptophan, respectively. SAMHD1 has deoxynucleoside triphosphate (dNTPase) activity and reduces cellular dNTP levels to restrict viral replication. These enzymes deplete the metabolic building blocks that are required for optimal viral replication and multiplication (32). SARSCOV-2 was more potent than SARS-CoV-1 in the induction of several interferon-responsive genes (25). In contrast, SAMHD1 was equally induced by SARS-CoV1 and SARS-C0V-2 (Figure 4B). The temporal induction of IDO1 was correlated with IFN-beta (IFNB1), STAT1, STAT2, and IRF9 at RNA levels (Figure 5A). These results are consistent with the interpretation that IFN- $\beta$ production by viral RNA recognition via Toll-like receptor (TLR) pathway drives autocrine or paracrine IFN- $\beta$ signaling involving the Jak-Stat pathway $(33,34)$. Productive infection of human lung epithelial cells by SARS CoV-2 requires cell membrane proteins ACE2 and TMPRSS2 to facilitate viral entry (35). SARS-CoV-2 infection of A549 cells failed to induce IFNB1 and interferon-stimulated gene expression. Expression of ACE2 in A549 cells restored SARS-CoV-2 -mediated induction of IFNB1, STAT1, STAT2, and IRF9 mRNA. Furthermore, gene expression of metabolic enzymes IDO1, SAT1, and SAMHD1 was restored in A549/ACE2 cells (Figure 5B). These studies revealed the important role of ACE2 in viral entry, activation of type I IFN signaling and temporal regulation of interferon-stimulated gene expression. Interrogation of the microarray dataset of HAE1 human lung epithelial cells revealed that IDOI induction peaked around 72 hours post-infection and declined by 96 hours in response to SARS-CoV-1. In contrast, IDO1 RNA levels gradually increased over 48 hours in response to MERS-CoV. These studies indicate 
that the temporal regulation of IDO1 RNA levels may vary depending on the type of the coronavirus and the lung cell line used in infection (Figure 6).

\subsection{Regulation of IDO1 expression by influenza and other respiratory viruses}

Influenza virus is a major respiratory pathogen that often causes significant mortality and morbidity, especially among young children and geriatric patients (36). Influenza is generally limited to the upper respiratory tract, but when the lower respiratory tract becomes involved, significant lung damage was observed. This may occur with pandemic strains (such as $1918 \mathrm{H} 1 \mathrm{~N} 1$ virus that killed more than 50 million worldwide) or pathogenic influenza strains (such as H5N1). Highly pathogenic strains of influenza such as the reconstructed 1918 influenza virus (H1N1) induced a dramatic increase in inflammatory cytokines, influx of neutrophils and macrophages resulting in inflammation, tissue injury and death in mice $(37,38)$. In contrast, seasonal non-pandemic strains of influenza inhibit type I interferon and inflammatory responses. In these strains, the virus encodes a non-structural protein (NS1) that is a potent inhibitor of Interferon regulatory factor 3 (IRF3) and type I interferon production (39). Furthermore, NS1 inhibits STAT1 activation resulting in the attenuation of interferon-stimulated gene expression (40). Induction of IDO1 expression in NHBE bronchial epithelial cells was abrogated by wild-type influenza A virus (IAV). In contrast, IAV infection with a deleted NS1 (IAVNS1) resulted in the induction of IDO1 expression in NHBE cells (Figure 7A). This induction of IDO1 was correlated with the induction of IFN-beta (IFNB1) and interferon-inducible transcription factor RNA of STAT1,

STAT2, IRF9 and IRF7 (Figure 7B). Furthermore, IAV mutant virus with NS1 deletion also induced metabolic gene expression of $\mathrm{CH} 25 \mathrm{H}$ and SAMHD1 in NHBE cells (Figure 7B). Infection of mice with the reconstructed 1918 influenza virus dramatically increased the cytokines TNF- $\alpha$ and IFN- $\gamma$, and antiviral and inflammatory gene expression (37). These include well-known interferon-stimulated genes (ISG) such as Guanylate binding protein (Gbp2) Interferon induced protein with tetratricopeptide 2 (Ifit2) 
and Ido1 (Figure 7C). Respiratory syncytial virus (RSV) infections are common in children and not a concern for adults or pose a pandemic threat. RSV infection also induced interferon-regulated metabolic enzymes IDO1 and SAMHD1 expression in A549 cells (Figure 8A and 8B). These studies suggest that multiple factors such as virus strains, host-encoded factors (ACE2), virus-encoded factors (NS1), and cell type play an important role in determining viral pathogenesis. A snapshot summary of IDO1 regulation by influenza and coronaviruses was shown (Figure 9). The red and blue ovals represented induction and suppression in separate microarray experiments, respectively. SAMHD1 was induced by cytokines such as IL-6, IFN- $\alpha / \beta$, IFN- $\gamma$, TLR-ligands such as LPS, and respiratory viruses (Figure 10). These results are consistent with SAMHD1's role in controlling antiviral and inflammatory gene expression (41,42). Furthermore, Cholesterol-25-hydroxylase and Spermidine acetyltransferase were also regulated by TLR-ligands and respiratory viruses (Supplementary data).

\subsection{Regulation of IDO1 expression by interferons and proinflammatory cytokine signaling}

Pathogen pattern recognition by lung resident immune cells such as dendritic cells and macrophages plays an important role in innate immunity. These cells have a variety of pattern recognition receptors (PRRs) including Toll-like receptors (TLR), retinoic acid-inducible gene I (RIG-I)-like receptors (RLR), and Nod-like receptors (NLR) to sense molecular signatures associated with pathogens (43). These receptors and associated adaptor molecules trigger kinase cascades leading to the activation of transcription factors such as nuclear factor-kB (NF-kB) and Interferon regulatory factors (IRF3, IRF7) resulting in the production of antiviral cytokines such as type-I Interferons (IFN) and proinflammatory cytokines such as Tumor necrosis Factor- $\alpha$ (TNF- $\alpha$ ), Interleukin-1 $\beta$ (IL-1 $\beta$ ) and Interleukin-6 (IL-6). Multiplex cytokine assays revealed inflammatory cytokines and chemokines in the bronchoalveolar lavage fluid of COVID-19 patients (9). Natural killer (NK) cells produce type-II interferon known as IFN- $\gamma$ (34). These cytokines activate transcription factors of STAT, IRF. NF-KB, and AP1 families and 
stimulate expression of a wide variety of genes encoding antiviral proteins and chemokines in lung epithelial cells resulting in the activation of key regulators of innate immunity (33). In type I Interferon (IFN- $\alpha / \beta$ ) signaling STAT1, STAT2, and IRF-9 transcription factor complexes (ISGF3) bind to interferon-stimulated response element (ISRE) in the gene promoter region to regulate gene expression. In contrast, STAT1 homodimers are predominantly activated and bind to gamma-activated sequence (GAS) in the gene promoter region to regulate IFN- $\gamma$-mediated gene expression. Furthermore, inducible transcription factor activation at RNA level enforces temporal regulation in both type-I and type-II IFN signaling (26). Interrogation of microarray datasets revealed that IFN- $\alpha / \beta$ treatment induced IDO1 and interferon-induced transcription factor at the RNA levels in 8 hours in A549 and HTBE human lung epithelial cells (Figure 11A and 11B). Furthermore, IFN- $\alpha$ induced IDO1 RNA by 8 to 10 -fold whereas IFN- $\gamma$ dramatically up-regulated by several hundred-fold within 12 to 24 hours in A549 cells (Figure 11C and 11D). Differential regulation of IDO1 by type-I and type-II interferons in multiple cell lines was reported in the Interferome database. Similarly, low and high induction of IDO1 by TLR ligands such as bacterial LPS and PAM3CSK4 was observed (Supplementary data). These studies reveal differential intensity of signaling mediating a dynamic range of IDO1 RNA fold-induction. A major limitation of these studies is the use of transformed or cancer cell lines. Immgen data on the effect of interferon treatment of primary immune cells such as dendritic cells and macrophages revealed that IFN- $\gamma$ was more potent than IFN- $\alpha$ in the induction of IDO1 RNA levels within 2 hours (data not shown). Cross-talk between cytokine signal transduction pathways enables the fine-tuning of the host response to different pathogens. There are two major outcomes in a cross-talk between the signal transduction pathwayseither transcriptional synergy or a functional antagonism $(44,45)$. For example, in mouse bone marrowderived macrophages, LPS and IFN- $\gamma$ synergistically induced IDO1 RNA levels (Figure 12A). In contrast, hypertonic saline solution (HTS) abrogated the induction of chemokines and inflammatory 
markers by a cytokine mixture of TNF- $\alpha$, IL-1 $\beta$, and IFN- $\gamma$ in human lung epithelial cells (46). HTS also abrogated IDO1 induction by proinflammatory cytokine mix in these cells (Figure 12B). These studies suggest that IDO1 RNA levels were differentially regulated in response to type I and type II interferons and inflammatory cytokines in human lung epithelial cells. Transcription factor binding site analysis in the promoter region of human IDO1 gene revealed GAS and ISRE elements as well as CCAAT enhancer-binding protein (CEBP- $\beta$ ) sequences located within the $1.3 \mathrm{~Kb}$ upstream of the transcription start site (Figure 12C). Nuclear content of STAT1 and IRF1 was significantly increased following cotreatment of IFN- $\gamma$ and TNF- $\alpha$, compared with treatment with either cytokine in Hela cells. TNF- $\alpha$ alone or in combination with IFN- $\gamma$ induced C/EBP- $\beta$ in Hela cells $(47,48)$. Gene promoter-luciferase reporter assays and electrophoretic mobility shift assay (EMSA) confirmed the role of these regulatory elements in mediating the synergistic activation of human IDO1 in response to IFN- $\gamma$ and TNF- $\alpha(47,48)$.

\subsection{Visualization of Protein interaction network of IDO1 in STRING database}

Protein interactions mediate post-translational modifications such as phosphorylation that activate or inhibit signaling and provide novel insights into the biological functions. These interactions can be visualized as a graph using the information in databases such as BIOGRID and STRING (28). Protein interactions of IDO1 interrogated in the STRING database revealed extensive connections to enzymes involved in tryptophan metabolism such as tryptophan 2,3-dioxygenase 2 (TDO2), neurotransmitter synthesis such as Monoamine oxidase A (MAOA), and Dopamine decarboxylase (DDC) as well as cytochrome P450 enzymes CYP1A1 and CYP1B1 involved in drug metabolism (Figure 13A). MAOA is located in the outer mitochondrial membrane and catalyzes the oxidative deamination of biogenic and xenobiotic amines (49). DDC, also known as aromatic-L-amino acid decarboxylase catalyzes the decarboxylation of L3,4-dihydroxyphenylalanine (L-DOPA) to dopamine (50). Human protein atlas (HPA) cell-specific RNA data revealed the co-expression of MAOA, DDC, CYP1A1, CYP1B1 enzymes 
with IDO1 in club cells revealing a potential for functional interactions (data not shown). Furthermore, IDO1 interacts with Suppressors of cytokine signaling 3 (SOCS3), STAT1, and STAT3 (Figure 13B). These interactions may play a major role in the transcriptional regulation of interferon and interleukin signaling (51-53).

\subsection{Regulation of IDO1 in Healthy and COVID-19 patients}

The innate immune response to a pathogenic virus infection must be controlled precisely and any imbalance in cytokine response may result in lung injury and death $(3,54)$. Previous studies have revealed differential regulation of several AT2-specific genes such as surfactant proteins (SFTPA1, SFTPA2, SFTPB, and SFTPC), and transport proteins (LAMP3 and SLC34A2) in the COVID-19 lung tissues (55). Interrogation of microarray datasets revealed that AT1 markers such as Advanced glycosylation endproduct specific receptor (AGER) and Epithelial membrane protein 2 (EMP2), ciliate cell marker Sentan, ciliary apical structure protein (SNTN), and club cell marker Secretoglobin family 1 member1 (SCGB1A1) were differentially regulated in the lung tissue of healthy and COVID-19 patients (Figure 14A). It is interesting to note that many of these proteins were identified as secreted proteins (surfactant proteins, SCGB1A1) or located in the plasma membrane (SLC34A2, LAMP3, EMP2, and AGER) suggesting the possibility of extracellular interactions between the proteins leading to altered biological functions in COVID-19 patients (Figure 14B). Furthermore, RNA levels of IDO1 and enzymes highly expressed in the club cells such as Aldehyde dehydrogenase 3 family member A1 (ALDH3A1) and Glutathione peroxidase 2 (GPX2) were significantly increased in the lung tissue of COVID-19 patients in comparison with healthy controls (Figure 14A). These results are consistent with the well-known role of club cells in drug metabolism and detoxification (30). A mix of proinflammatory cytokines such as IFN$\gamma$, TNF- $\alpha$, and IL-1 $\beta$ induced a dramatic increase in cytokines and chemokines in the lung epithelial cells in cell-culture (46). The cytokine mix-induced gene expression patterns were similar to the 
transcriptomic changes in the lung tissue of COVID-19 patients (Figure 15A and 15B). Interestingly, hypertonic saline solution (HTS) or hyperosmolarity prevented the cytokine and chemokine expression in the cell culture model. One notable exception is the further enhancement of cytokine mix-induced Platelet-derived growth factor receptor alpha (PDGFRA) gene expression by HTS treatment. Tyrosine and serine kinases play a major role in the growth factor and cytokine-mediated signal transduction in eukaryotic cells. Intracellular phosphorylation and dephosphorylation of signal transduction proteins provide a rapid switch to modulate and fine-tune gene expression (56--58). Several kinases involved in interferon (JAK2, EIF2AK2), TNF- $\alpha$ (RIPK2), IL-1 $\beta$ (IRAK3) signaling were up-regulated by cytokine mix in the lung epithelial cells in cell culture and in COVID-19 patients. (Figure 16A and 16B). Furthermore, HTS treatment attenuated the induction of genes encoding kinases in the lung epithelial cells in cell culture (Figure 16A). These studies are consistent with the results suggesting osmolarity regulates gene expression by rapidly modulating signaling and transcription networks $(46,59)$.

\section{Conclusion}

Metabolic profiling of serum samples from COVID-19 patients revealed alterations of cholesterol and tryptophan metabolic pathways $(11,12)$. Interferon-stimulated genes (ISG) encoding catabolic enzymes such as IDO1, CH25H, SAT1, and SAMHD1 deplete the metabolic building blocks such as essential amino acid, lipid, polyamine, and nucleotide pools to restrict viral replication and multiplication (32). IDO1 was induced by interferons and SARS-CoV-2 in human lung epithelial cells. Consistent with these results, IDO1 RNA levels were up-regulated in the lung tissue of COVID-19 patients in comparison with healthy individuals. The induction of IDO1 RNA by respiratory viruses in lung epithelial cells in cell culture was correlated with the induction of IFNB1 and IFN-induced transcription factor RNA levels. Furthermore, a potent mix of proinflammatory cytokines IFN- $\gamma$, TNF- $\alpha$, and IL- $1 \beta$ dramatically induced IDO1 RNA levels in lung cells. These results suggest that both direct effects of the virus, as well as host 
immune responses, contribute to high levels of IDO1 RNA levels. Genetic variability in different individuals leads to different clinical courses and treatment responses to COVID-19. Genetic analysis revealed that about $10 \%$ critically ill COVID-19 patients have alterations in the components of type-I interferon signaling or autoantibodies to interferon that inactivate antiviral signaling (60-62). Furthermore, gene expression profiling of nasal swabs revealed that mild or recovered COVID -19 has a robust type I interferon response in the nasopharynx than critically ill patients (63). These results suggest that type I interferon signaling is important for innate immunity to SARS-CoV-2 infection and of diagnostic value for predicting the progression of the disease. However, the efficacy of antiviral drugs such as remidisvir, hydroxychloroquine, and interferon for the treatment of COVID-19 patients remains controversial (64). In contrast, treatment with anti-inflammatory dexamethasone has proven to be therapeutically beneficial to COVID-19 patients (65). The emergence of SARS-CoV-2 variants such as delta with increased viral replication and pathogenicity in the human populations is of major concern. There is a need to develop novel strategies to down-regulate the inflammatory response in COVID-19 patients. IDO1 inhibitors are in clinical trials either as monotherapy or in combination with other therapies for cancer treatment $(66,67)$. Whether the IDO1 inhibitor treatment alone or in combination with dexamethasone to dampen inflammation has any beneficial effects in COVID-19 patients remains to be determined.

\section{References}

1. Ko JY, Danielson ML, Town M, Derado G, et. al. COVID-NET Surveillance Team. Risk Factors for Coronavirus Disease 2019 (COVID-19)-Associated Hospitalization: COVID-19-Associated Hospitalization Surveillance Network and Behavioral Risk Factor Surveillance System. Clin Infect Dis. 2021 Jun 1;72(11):e695-e703 
2. Grasselli G, Tonetti T, Protti A, Langer, T, et. al Pathophysiology of COVID-19-associated acute respiratory distress syndrome: a multicentre prospective observational study. Lancet Respir Med. 2020 Dec;8(12):1201-1208. doi: 10.1016/S2213-2600(20)30370-2.

3. Blanco-Melo D, Nilsson-Payant BE, et. al. Imbalanced Host Response to SARS-CoV-2 Drives Development of COVID-19. Cell. 2020 May 28;181(5):1036-1045.e9.

4. Xu J, Xu X, Jiang L, Dua K, Hansbro PM, Liu G. SARS-CoV-2 induces transcriptional signatures in human lung epithelial cells that promote lung fibrosis. Respir Res. 2020 Jul 14;21(1):182.

5. Bojkova D, Klann K, Koch B, Widera M, Krause D, Ciesek S, Cinatl J, Münch C. Proteomics of SARS-CoV-2-infected host cells reveals therapy targets. Nature. 2020 Jul;583(7816):469-472. doi: $10.1038 / \mathrm{s} 41586-020-2332-7$.

6. Gordon, D. E. et al. A SARS-CoV-2-Human Protein-Protein Interaction Map Reveals Drug Targets and Potential Drug-Repurposing. bioRxiv, 2020. doi:10.1101/2020.03.22.002386.

7. Ziegler CGK, Allon SJ, Nyquist SK,. Et.al. SARS-CoV-2 Receptor ACE2 Is an InterferonStimulated Gene in Human Airway Epithelial Cells and Is Detected in Specific Cell Subsets across Tissues. Cell. 2020 May 28;181(5):1016-1035.e19. doi: 10.1016/j.cell.2020.04.035.

8. Delorey TM, Ziegler CGK, Heimberg G, et.al. COVID-19 tissue atlases reveal SARS-CoV-2 pathology and cellular targets. Nature. 2021 Jul;595(7865):107-113. doi: 10.1038/s41586-02103570-

9. Lucas C, Wong P, Klein J, Castro TBR, et.al. Longitudinal analyses reveal immunological misfiring in severe COVID-19. Nature. 2020; 584:463-469.

10. Stukalov A, Girault V, Grass V, et. al. Multilevel proteomics reveals host perturbations by SARS-CoV-2 and SARS-CoV. Nature. 2021 Jun;594(7862):246-252. doi: 10.1038/s41586-02103493-4. 
11. Thomas T, Stefanoni D, Reisz JA, et. al. COVID-19 infection alters kynurenine and fatty acid metabolism, correlating with IL-6 levels and renal status. JCI Insight. 2020 Jul23;5(14):e140327. doi: 10.1172/jci.insight.140327.

12. Lionetto L, Ulivieri M, Capi M,et. al. Increased kynurenine-to-tryptophan ratio in the serum of patients infected with SARS-CoV2: An observational cohort study. Biochim Biophys Acta Mol Basis Dis. 2021 Mar 1;1867(3):166042. doi: 10.1016/j.bbadis.2020.166042.

13. Prendergast GC, Malachowski WJ, Mondal A, Scherle P, Muller AJ. Indoleamine 2,3Dioxygenase and Its Therapeutic Inhibition in Cancer. Int Rev Cell Mol Biol. 2018;336:175-203. doi: 10.1016/bs.ircmb.2017.07.004

14. Meireson A, Devos M, Brochez L. IDO Expression in Cancer: Different Compartment, Different Functionality? Front Immunol. 2020 Sep 24;11:531491. doi: 10.3389/fimmu.2020.531491.

15. Prendergast GC, Malachowski WP, DuHadaway JB, Muller AJ. Discovery of IDO1 inhibitors: from bench to bedside. Cancer Res. (2017) 77:6795- 811.

16. Hassanain HH, Chon SY, Gupta SL. Differential regulation of human indoleamine 2,3dioxygenase gene expression by interferons-gamma and -alpha. Analysis of the regulatory region of the gene and identification of an interferon-gamma-inducible DNA-binding factor. J Biol Chem. (1993) 268:5077-84.

17. Mailankot M, Nagaraj RH. Induction of indoleamine 2,3-dioxygenase by interferon-gamma in human lens epithelial cells: apoptosis through the formation of 3-hydroxykynurenine. Int J Biochem Cell Biol. 2010 Sep;42(9):1446-54. doi: 10.1016/j.biocel.2010.04.014.

18. Babcock TA, Carlin JM. Transcriptional activation of indoleamine dioxygenase by interleukin 1 and tumor necrosis factor alpha in interferon-treated epithelial cells. Cytokine. (2000) 12:588-94. doi: 10.1006/cyto.1999.0661 2 
19. Litzenburger UM, Opitz CA, Sahm F, Rauschenbach KJ, Trump S, Winter M, et al. Constitutive IDO expression in human cancer is sustained by an autocrine signaling loop involving IL-6, STAT3 and the AHR. Oncotarget. (2014) 5:1038-51.

20. Yusufu I, Ding K, Smith K, Wankhade UD, Sahay B, Patterson GT, Pacholczyk R, Adusumilli S, Hamrick MW, Hill WD, Isales CM, Fulzele S. A Tryptophan-Deficient Diet Induces Gut Microbiota Dysbiosis and Increases Systemic Inflammation in Aged Mice. Int J Mol Sci. 2021 May 8;22(9):5005. doi: 10.3390/ijms22095005.

21. Fox JM, Crabtree JM, Sage LK, Tompkins SM, Tripp RA. Interferon Lambda Upregulates IDO1 Expression in Respiratory Epithelial Cells After Influenza Virus Infection. J Interferon Cytokine Res. 2015 Jul;35(7):554-62. doi: 10.1089/jir.2014.0052.

22. Pfefferkorn ER. Interferon gamma blocks the growth of Toxoplasma gondii in human fibroblasts by inducing the host cells to degrade tryptophan. Proc Natl Acad Sci USA. (1984) 81:908-12.

23. Baranyi A, Meinitzer A, Breitenecker RJ, Amouzadeh-Ghadikolai O, Stauber R, Rothenhäusler HB. Quinolinic Acid Responses during Interferon- $\alpha$-Induced Depressive Symptomatology in Patients with Chronic Hepatitis C Infection - A Novel Aspect for Depression and Inflammatory Hypothesis. PLoS One. 2015 Sep 14;10(9):e0137022.

24. Bosco MC, Rapisarda A, Reffo G, Massazza S, Pastorino S, Varesio L. Macrophage activating properties of the tryptophan catabolite picolinic acid. Adv Exp Med Biol. 2003;527:55-65.

25. Ramana C.V. Regulation of early growth response 1 (Egr-1) gene expression by Stat1 independent type I interferon signaling and respiratory viruses. bioRxiv (2020). https://doi.org/10.1101/2020.08.14.244897 
26. Ramana C.V. Profiling transcription factor sub-networks in type I interferon signaling and in response to SARS-CoV-2 infection. bioRxiv (2021); DOI: 10.1101/2021.01.25.428122. https://doi.org/10.1101/2021.01.25.428122

27. Babicki S, Arndt D, Marcu A, Liang Y, Grant JR, Maciejewski A, Wishart DS. Heatmapper: web- enabled heat mapping for all. Nucleic Acids Res. 2016; 44(W1):W147-53.

28. Szklarczyk D, Morris JH, Cook H, Kuhn M, Wyder S, Simonovic M, Santos A, Doncheva NT, Roth A, Bork P, Jensen LJ, von Mering C. The STRING database in 2017. Quality-controlled protein-protein association networks, made broadly accessible. Nucleic Acids Res. 2017; 45:D362D368.

29. Zhou Y, Zhou B, Pache L, Chang M, Khodabakhshi AH, Tanaseichuk O, Benner C, Chanda SK. Metascape provides a biologist-oriented resource for the analysis of systems-level datasets. Nat Commun. 2019; 10:1523.

30. Rokicki W, Rokicki M, Wojtacha J, Dżeljijli A. The role and importance of club cells (Clara cells) in the pathogenesis of some respiratory diseases. Kardiochir Torakochirurgia Pol. 2016 Mar;13(1):26-30. doi: 10.5114/kitp.2016.58961.

31. Agliardi C, Guerini FR, Zanzottera M, Rovaris M, Caputo D, Clerici M. Indoleamine-2,3dioxygenase(IDO)2 polymorphisms are not associated with multiple sclerosis in Italians. J Neurol Sci. 2017 Jun 15;377:31-34. doi: 10.1016/j.jns.2017.03.048.

32. Raniga K, Liang C. Interferons: Reprogramming the Metabolic Network against Viral Infection. Viruses. 2018 Jan 13;10(1):36. doi: 10.3390/v10010036.

33. Kumar H, Kawai T, Akira S. Pathogen recognition by the innate immune system. Int Rev Immunol. 2011; 30(1):16-34. 
34. Ardain A, Marakalala MJ, Leslie A. Tissue-resident innate immunity in the lung. Immunology. $2020 ; 159: 245-256$.

35. Wang A, Chiou J, Poirion OB, Buchanan J, Valdez MJ, Verheyden JM, Hou X, Kudtarkar P, Narendra S, Newsome JM, Guo M, Faddah DA, Zhang K, Young RE, Barr J, Sajti E, Misra R, Huyck H, Rogers L, Poole C, Whitsett JA, Pryhuber G, Xu Y, Gaulton KJ, Preissl S, Sun X; NHLBI LungMap Consortium. Single-cell multi omic profiling of human lungs reveals cell-type-specific and age-dynamic control of SARS-CoV2 host genes. Elife. 2020; 9:e62522.

36. Michael P, Brabant D, Bleiblo F, Ramana CV, Rutherford M, Khurana S, Tai TC, Kumar A, Kumar A. Influenza A induced cellular signal transduction pathways. J Thorac Dis. 2013;5 Suppl 2:S132-41.

37. Kash JC, Tumpey TM, Proll SC, et al. Genomic analysis of increased host immune response and cell death responses induced by 1918 influenza virus. Nature. 2008, 443: 578-581.

38. Julkunen I, Sareneva T Pirhonen, T et al. Molecular pathogenesis of influenza A virus infection and virus-induced regulation of gene expression. Cytokine growth factor Rev. 2001, 12: 171-180. 39. Gack MU, Albrecht RA, Urano T, Inn KS, Huang IC, Carnero E, et al. Influenza A virus NS1 targets the ubiquitin ligase TRIM25 to evade recognition by the host viral RNA sensor RIG-I. Cell Host Microbe. 2009; 5: 439-449.

40. Han CW, Jeong MS, Jang SB. Structure and Function of the Influenza A Virus Non-Structural Protein 1. J Microbiol Biotechnol. 2019; 29(8):1184-1192.

41. Chen S, Bonifati S, Qin Z, St Gelais C, Wu L. SAMHD1 Suppression of Antiviral Immune Responses. Trends Microbiol. 2019 Mar;27(3):254-267. doi: 10.1016/j.tim.2018.09.009. Epub 2018 Oct 15. PMID: 30336972; PMCID: PMC6377309. 
42. Sze A, Olagnier D, Lin R, van Grevenynghe J, Hiscott J. SAMHD1 host restriction factor: a link with innate immune sensing of retrovirus infection. J Mol Biol. 2013 Dec 13;425(24):4981-94. doi: 10.1016/j.jmb.2013.10.022.

43. Beutler B. Microbe sensing, positive feedback loops, and the pathogenesis of inflammatory diseases. Immunol Rev. 2009 Jan;227(1):248-63. doi: 10.1111/j.1600-065X.2008.00733.x.

44. Ramana CV, Chatterjee-Kishore M, Nguyen H, Stark GR. Complex roles of Stat1 in regulating gene expression. Oncogene. 2000 May 15;19(21):2619-27. doi: 10.1038/sj.onc.1203525. PMID: 10851061.

45. Adelaja A, Hoffmann A. Signaling Crosstalk Mechanisms That May Fine-Tune PathogenResponsive NFkB. Front Immunol. 2019; 10:433.

46. Mitra S, Schiller D, Anderson C, Gamboni F, D'Alessandro A, Kelher M, Silliman CC, Banerjee A, Jones KL. Hypertonic saline attenuates the cytokine-induced pro-inflammatory signature in primary human lung epithelia. PLoS One. 2017 Dec 18;12(12):e0189536. doi: 10.1371/journal.pone.0189536. PMID: 29253007; PMCID: PMC5734749.

47. Robinson, CM., Shirley, KA., and Carlin, J.M. (2003). Synergistic transcriptional activation of indoleamine dioxygenase by IFN-gamma and tumor necrosis factor-alpha. J. Interferon Cytokine Res. 23, 413-421.

48. Robinson CM, Hale PT, Carlin JM. The role of IFN- $\gamma$ and TNF- $\alpha$-responsive regulatory elements in the synergistic induction of indoleamine dioxygenase. J Interferon Cytokine Res. (2005) 25:20-30. doi: 10.1089/jir.2005.25.20 20.

49. Benedetti MS. Biotransformation of xenobiotics by amine oxidases. Fundam Clin Pharmacol. 2001 Apr;15(2):75-84 
50. Hodgetts RB, O'Keefe SL. Dopa decarboxylase: a model gene-enzyme system for studying development, behavior, and systematics. Annu Rev Entomol. 2006;51:259-84.

51. Gao Y, Zhao H, Wang P, Wang J, Zou L. The roles of SOCS3 and STAT3 in bacterial infection and inflammatory diseases. Scand J Immunol. 2018 Dec;88(6):e12727.

52. Kang S, Tanaka T, Narazaki M, Kishimoto T. Targeting Interleukin-6 Signaling in Clinic. Immunity. 2019 Apr 16;50(4):1007-1023.

53. Kile BT, Alexander WS. The suppressors of cytokine signalling (SOCS). Cell Mol Life Sci. 2001 Oct;58(11):1627-35.

54. Arunachalam PS, Wimmers F, Mok CKP, et.. al Systems biological assessment of immunity to mild versus severe COVID-19 infection in humans. Science. 2020; 369:1210-1220.

55. Ramana, C.V. Regulation of Lysosome-Associated Membrane Protein 3 (LAMP3) in Lung Epithelial Cells by Coronaviruses (SARS-CoV-1/2) and Type I Interferon Signaling.. BioRxiv (2021); DOI: 10.1101/2021.04.28.441840.

56. Thorner J, Hunter T, Cantley LC, Sever R. Signal transduction: From the atomic age to the postgenomic era. Cold Spring Harb Perspect Biol. 2014 Oct 30;6(12):a022913.

57. Nguyen H, Ramana CV, Bayes J, Stark GR. Roles of phosphatidylinositol 3-kinase in interferongamma-dependent phosphorylation of STAT1 on serine 727 and activation of gene expression. J Biol Chem. 2001 Sep 7;276(36):33361-8. doi: 10.1074/jbc.M105070200. Epub 2001 Jul 3. PMID: 11438544.

58. Nguyen H, Chatterjee-Kishore M, Jiang Z, Qing Y, Ramana CV, Bayes J, Commane M, Li X, Stark GR. IRAK-dependent phosphorylation of Stat 1 on serine 727 in response to interleukin-1 and effects on gene expression. J Interferon Cytokine Res. 2003 Apr;23(4):183-92. 
59.Wright FL, Gamboni F, Moore EE, Nydam TL, Mitra S, Silliman CC, Banerjee A. Hyperosmolarity invokes distinct anti-inflammatory mechanisms in pulmonary epithelial cells: evidence from signaling and transcription layers. PLoS One. 2014 Dec 5;9(12):e114129.

60. Zhang Q, Bastard P, Liu Z, Le Pen J, Moncada-Velez M, Chen J, et.al. Inborn errors of type I IFN immunity in patients with life-threatening COVID-19. Science. 2020; 370:eabd4570

61. Bastard P, Rosen LB, Zhang Q, Michailidis E, Hoffmann HH, et. al. Autoantibodies against type I IFNs in patients with life-threatening COVID-19. Science. 2020; 370(6515)

62. Pairo-Castineira E, Clohisey S, Klaric L, Bretherick AD, Rawlik K,. Genetic mechanisms of critical illness in Covid-19. Nature. 2020. doi: 10.1038/s41586-020-03065-y.

63. Ziegler CGK, Miao VN, Owings AH, Navia AW, Tang Y, Bromley JD, et. al. Impaired local intrinsic immunity to SARS-CoV-2 infection in severe COVID-19. Cell. 2021 Jul 23:S00928674(21)00882-5. doi: 10.1016/j.cell.2021.07.023. Epub ahead of print. PMID: 34352228 64. Zhao M, Zhang J, Li H, Luo Z, Ye J, Xu Y, Wang Z, Ye D, Liu J, Li D, Wang M, Wan J. Recent progress of antiviral therapy for coronavirus disease 2019. Eur J Pharmacol. 2021 Jan 5;890:173646. doi: 10.1016/j.ejphar.2020.173646.

65. Noreen S, Maqbool I, Madni A. Dexamethasone: Therapeutic potential, risks, and future projection during COVID-19 pandemic. Eur J Pharmacol. 2021 Mar 5;894:173854. doi: 10.1016/j.ejphar.2021.173854.

66. Le Naour J, Galluzzi L, Zitvogel L, Kroemer G, Vacchelli E. Trial watch: IDO inhibitors in cancer therapy. Oncoimmunology. 2020 Jun 14;9(1):1777625. doi: 10.1080/2162402X.2020.1777625

67. Zhai L, Ladomersky E, Lenzen A, Nguyen B, Patel R, Lauing KL, Wu M, Wainwright DA. IDO1 in cancer: a Gemini of immune checkpoints. Cell Mol Immunol. 2018 May;15(5):447-457. 


\section{Figure Legends}

Figure 1. Expression levels of IDO1 RNA in human tissues in the GTEx database. High levels of IDO1 expression in the lungs, stomach and esophagus tissues were shown. RNA levels were expressed as transcripts per million reads or TPM.

Figure 2. Cell-specific expression of IDO1, IDO2 and SCGB1A1 RNA levels in the Human Protein Cell Atlas (HPA). RNA expression profiles were retrieved from the human lung tissue dataset of HPA. Human lung cell population of the c-1 and c-6 epithelial type II (AT2) and the c-7 club cells were indicated by an asterisk. RNA levels were expressed as transcripts per million reads or TPM.

Figure 3. Heatmap representation of the RNA levels of IDO1 in the distinct cell populations of the human lung. RNA expression profiles were retrieved from the Human Protein Atlas and represented as a heatmap. Human lung AT2 c-1, c-6 and club cell population were indicated by an asterisk. Expression levels of selected lung AT1, AT2, ciliate and club markers were shown for comparison.

Figure 4. Regulation of interferon-regulated metabolic enzymes at RNA levels by the SARS CoV-1 and SARS-CoV-2 in Calu-3 cells (A) Relative levels of IDO1 RNA after mock infection or virus infection in Calu-3 cells for 12 or 24 hours were shown (B) Heatmap representation of IFNregulated genes at RNA levels. Data from two samples for each condition were shown. RNA expression levels were normalized by the DEseq2 method.

Figure 5. Regulation of the interferon-stimulated transcription factors and IDO1 RNA levels by coronaviruses in Calu-3, A549 or A549 cells expressing ACE2 receptors (A) The expression levels of IFN-regulated transcription factors and IDO1 mRNA in Calu-3 cells after infection with SARSCoV-1 or SARS-CoV-2 for 4, 12, or 24 hours were shown (B) A549 or A549 cells expressing ACE2 receptors were infected with the SARS-CoV-2 virus for 24 hours and RNA fold-induction of 
IFN-regulated genes for each condition was shown. Data from three samples for each condition were shown.

Figure 6. Temporal regulation of IDO1 RNA induction by coronaviruses in HAE-1 cells. Relative levels of IDO1 RNA levels after infection with SARS-CoV-1 or MERS-CoV for 12 to 96 hours in HAE-1 cells. Log2 fold-change in IDO1 RNA expression levels were shown. HPI refers to hours post-infection.

Figure 7. Regulation of IDO1 RNA levels by the influenza viruses (A) Human NHBE1 bronchial epithelial cells were mock-infected or infected with the influenza virus (IAV) or influenza virus lacking the NS1 (IAVNS1) for 12 hours. Results from three samples for each condition were shown (B)) Heatmap representation of interferon-regulated gene expression regulated by mock, wild-type IAV and NS1 deleted IAV infection (C) Regulation of interferon-stimulated gene expression in mouse lungs after infection with reconstructed 1918 H1N1 influenza virus. Fold induction of Ido1, Gbp2 and Ifit2 were shown.

Figure 8. Regulation of IDO1 and SAMHD1 mRNA levels by the respiratory syncytial virus (A) Human A549 lung cells were mock-infected or infected with the RSV for 24 hours and average foldinduction of IDO1 RNA from three samples were shown. (B) A549 cells were mock-infected or infected with RSV for 24 hours. Data from 3 samples for each condition was shown. SAMHD1 mRNA levels were normalized by the DESeq2 method.

Figure 9. A snapshot of regulation of IDO1 mRNA levels by respiratory viruses as represented in the microarray datasets. Induction or suppression of IDO1 RNA levels were represented by red and blue ovals, respectively. Each oval represents a separate experiment. 
Figure 10. A snapshot of regulation of SAMHD1 RNA levels by cytokines, TLR ligands, respiratory viruses as represented in the microarray datasets. Induction or suppression of SAMHD1 RNA levels were represented by red and blue ovals, respectively. Each oval represents a separate experiment.

Figure 11. Regulation of the interferon-stimulated genes (ISG) at RNA levels by interferon treatment in A549 and HTBE human lung epithelial cells (A) Regulation of RNA levels of IDO1 and selected ISG by IFN- $\alpha / \beta$ treatment for 8 hours in A549 cells (B) Regulation of RNA levels of ISG by IFN$\alpha / \beta$ treatment for 8 hours in HTBE cells (C) Regulation of IDO11 RNA levels by IFN- $\alpha$ treatment for 12 and 24 hours in A549 cells (D) Regulation of IDO11 RNA levels by interferon-gamma IFN- $\gamma$ treatment for 12 and 24 hours in A549 cells. Gene expression data were retrieved from microarray datasets.

Figure 12. Cross-talk between proinflammatory signaling pathways in the regulation of IDO1 gene expression (A) Synergistic induction of IDO1 gene expression by LPS and IFN- $\gamma$ treatment in mouse bone marrow-derived macrophages (B) Functional antagonism between hypertonic saline solution (HTS) and proinflammatory cytokines in the regulation of IDO1 gene expression (C) Schematic representation of the human IDO1 gene promoter. The approximate location of the GAS, ISRE and CEBP sequence elements in the human IDO1 gene promoter were shown. TSS represents the transcription start site.

Figure 13. IDO1 and its protein-protein interaction (PPI) network. Interactions of IDO1 and other proteins involved in tryptophan metabolism, interleukin and Stat signaling were represented in the STRING database. Interacting proteins were indicated by ovals and protein interactions by edges, respectively.

Figure 14. Regulation of RNA levels of IDO1 and selected human lung cell-specific markers in healthy controls and COVID-19 patients (A) RNA expression levels of lung cell-specific markers in 
the healthy and COVID-19 lung samples were shown. Low and high expression in healthy and COVID-19 lung biopsies were represented by blue and red, respectively. RNA expression values were normalized by the DEseq2 method and shown as a heat map. Data from two samples for each condition were shown (B) Protein interactions of IDO1 with AT2, AT1 and club cell markers as represented in the STRING database.

Figure 15. Regulation of selected chemokines and inflammatory gene expression in the human lung epithelial cells and in the healthy and COVID-19 lung tissues (A) Cytokine mix mediated induction of inflammatory gene expression and abrogation by hypertonic saline solution (HTS) treatment in human lung epithelial cells were shown (B) Regulation of selected chemokines and inflammatory gene expression in the healthy and COVID-19 lung samples. Low and high expression in healthy and COVID-19 lung biopsies were represented by blue and red, respectively. Data from two samples for each condition were shown.

Figure 16. Regulation of selected kinases and inflammatory gene expression in the human lung epithelial cells and in the healthy and COVID-19 lung samples (A) Cytokine mix mediated induction of kinases and inflammatory gene expression and abrogation by hypertonic saline solution (HTS) treatment in human lung epithelial cells were shown (B) Regulation of selected kinases and inflammatory gene expression in the healthy and COVID-19 lung tissues. Low and high expression in healthy and COVID-19 lung biopsies were represented by blue and red, respectively. Data from two samples for each condition were shown. 


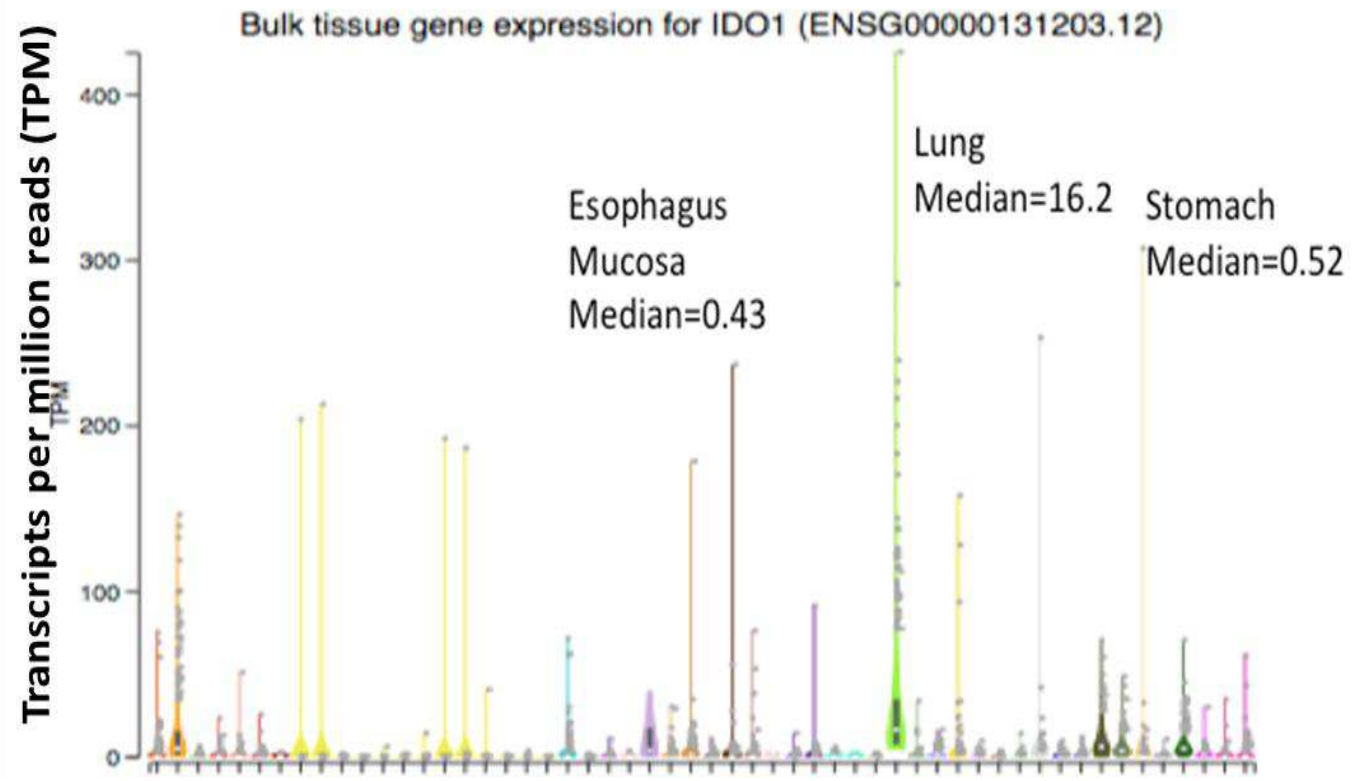

Human tissues

Figure 1 

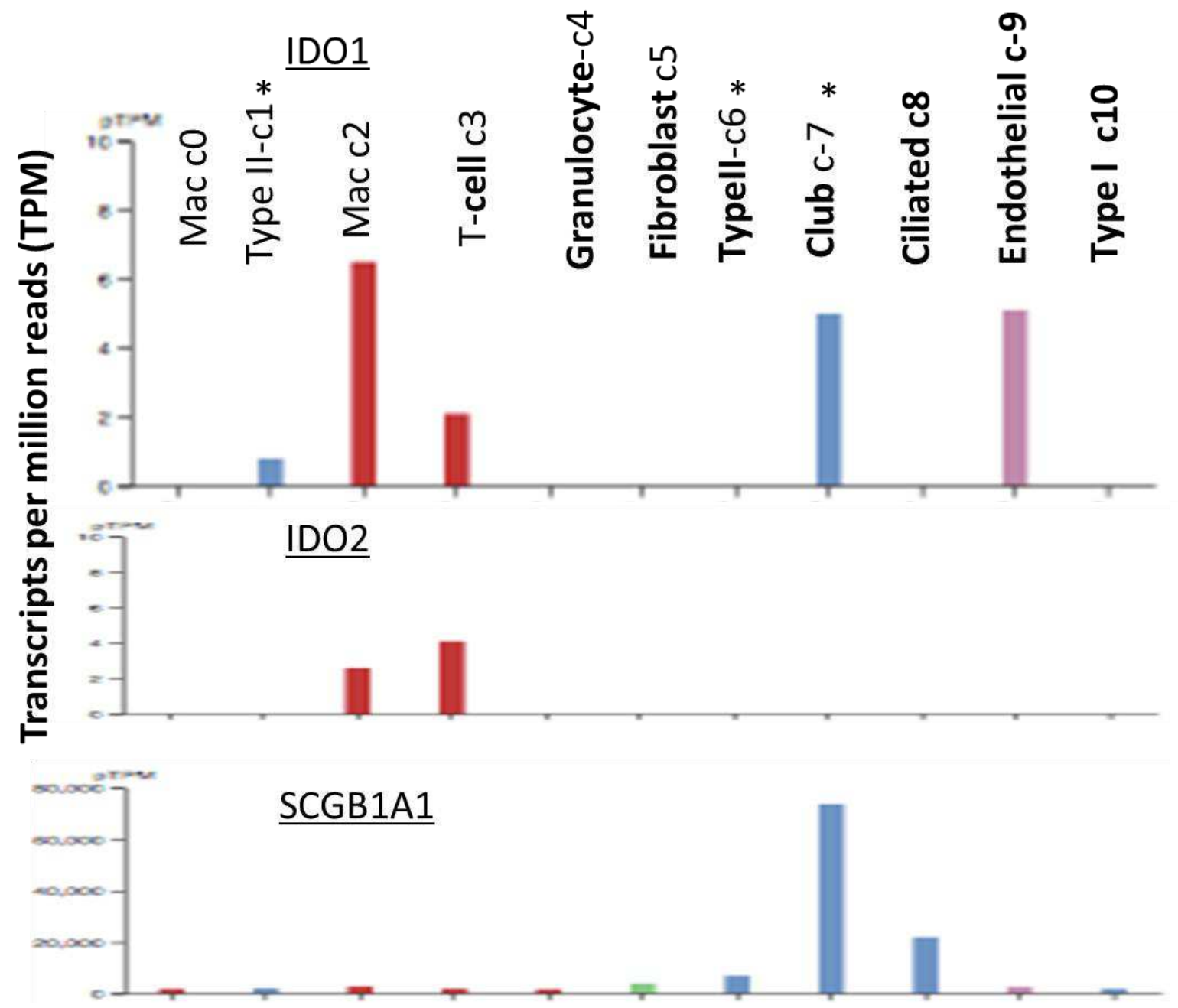

Figure 2 


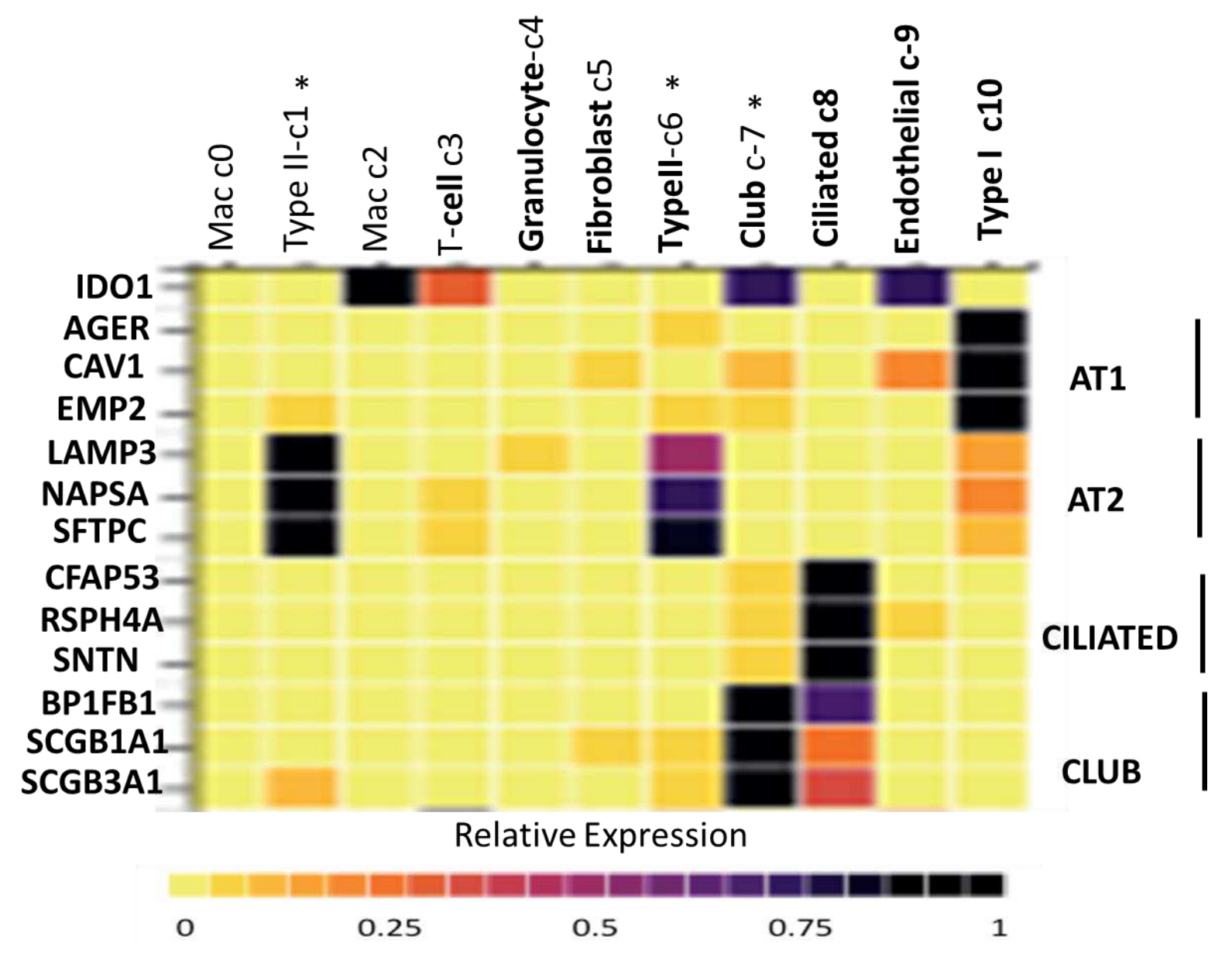

Figure 3 


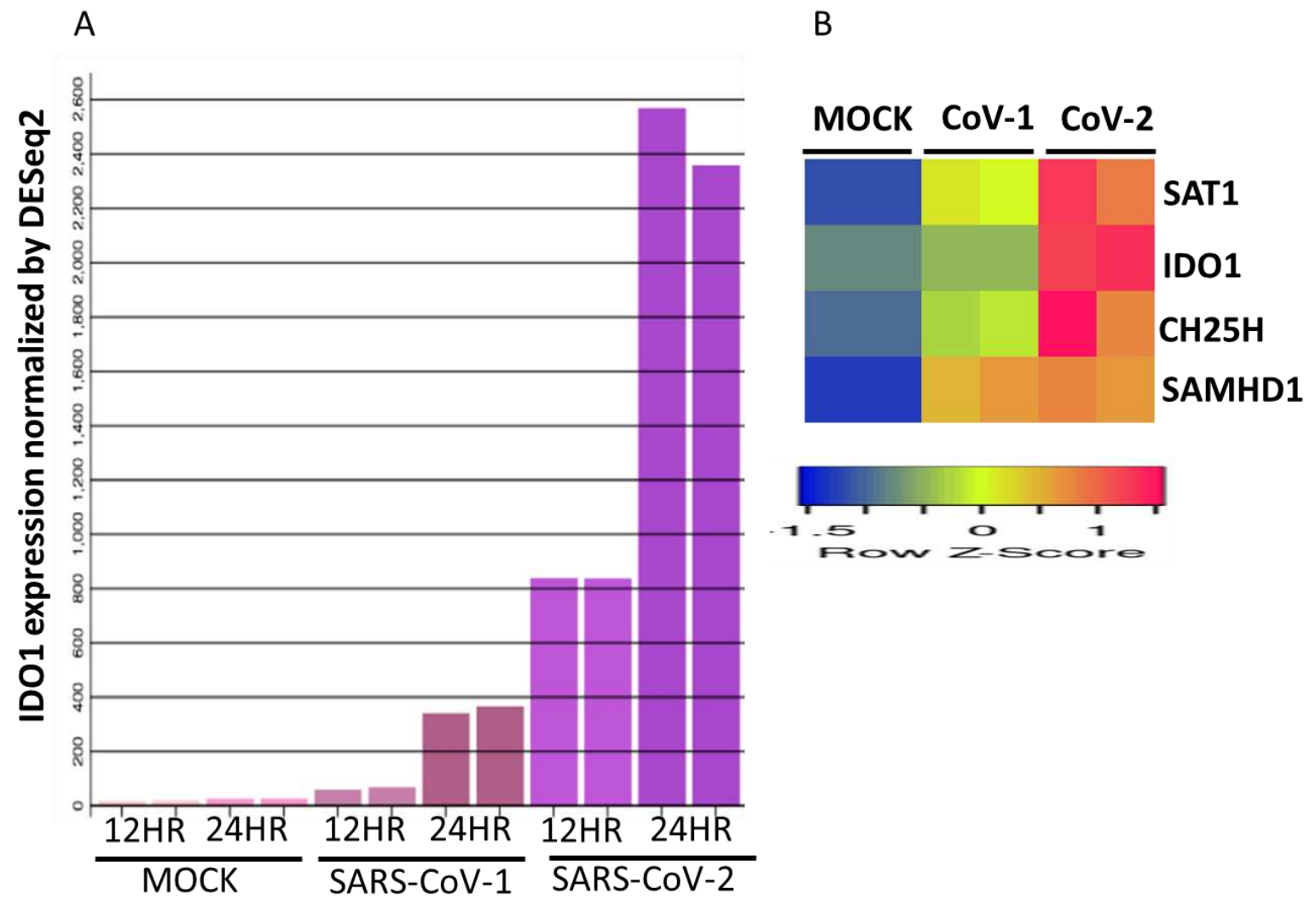

Figure 4 
A

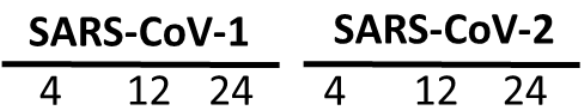

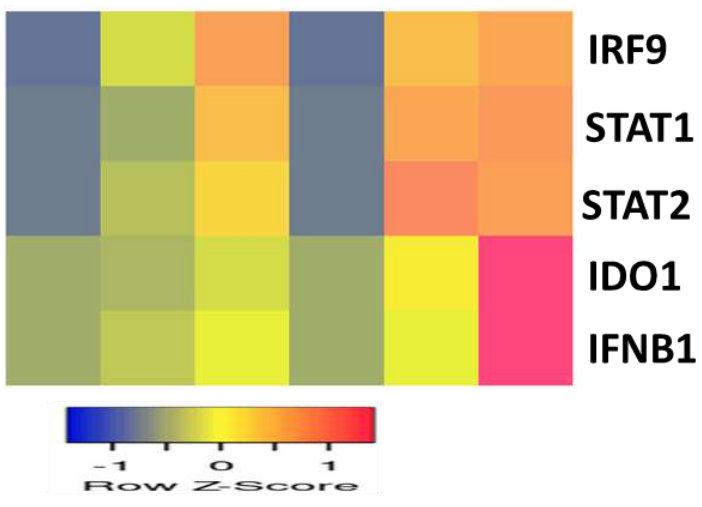

B

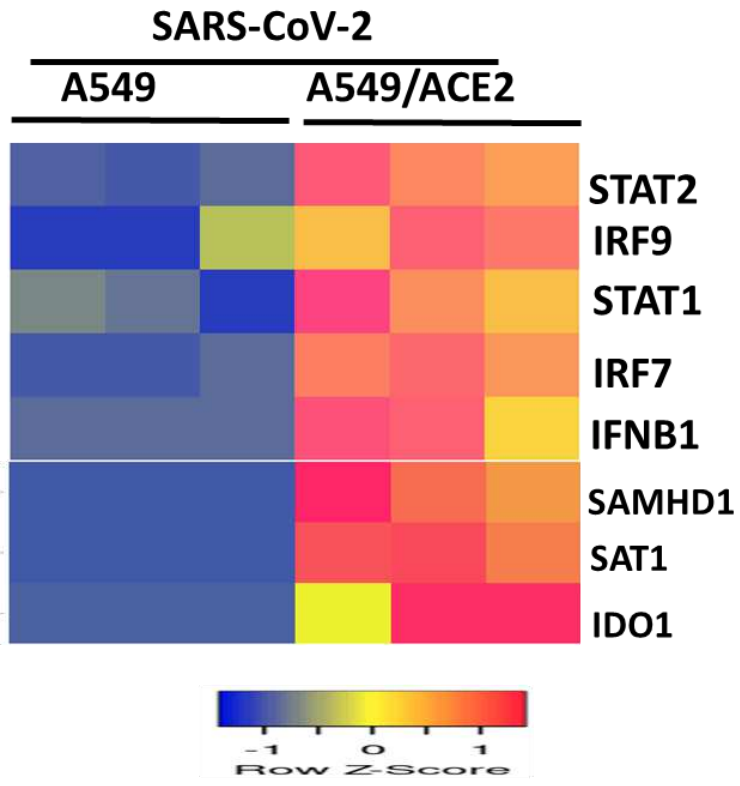

Figure 5 


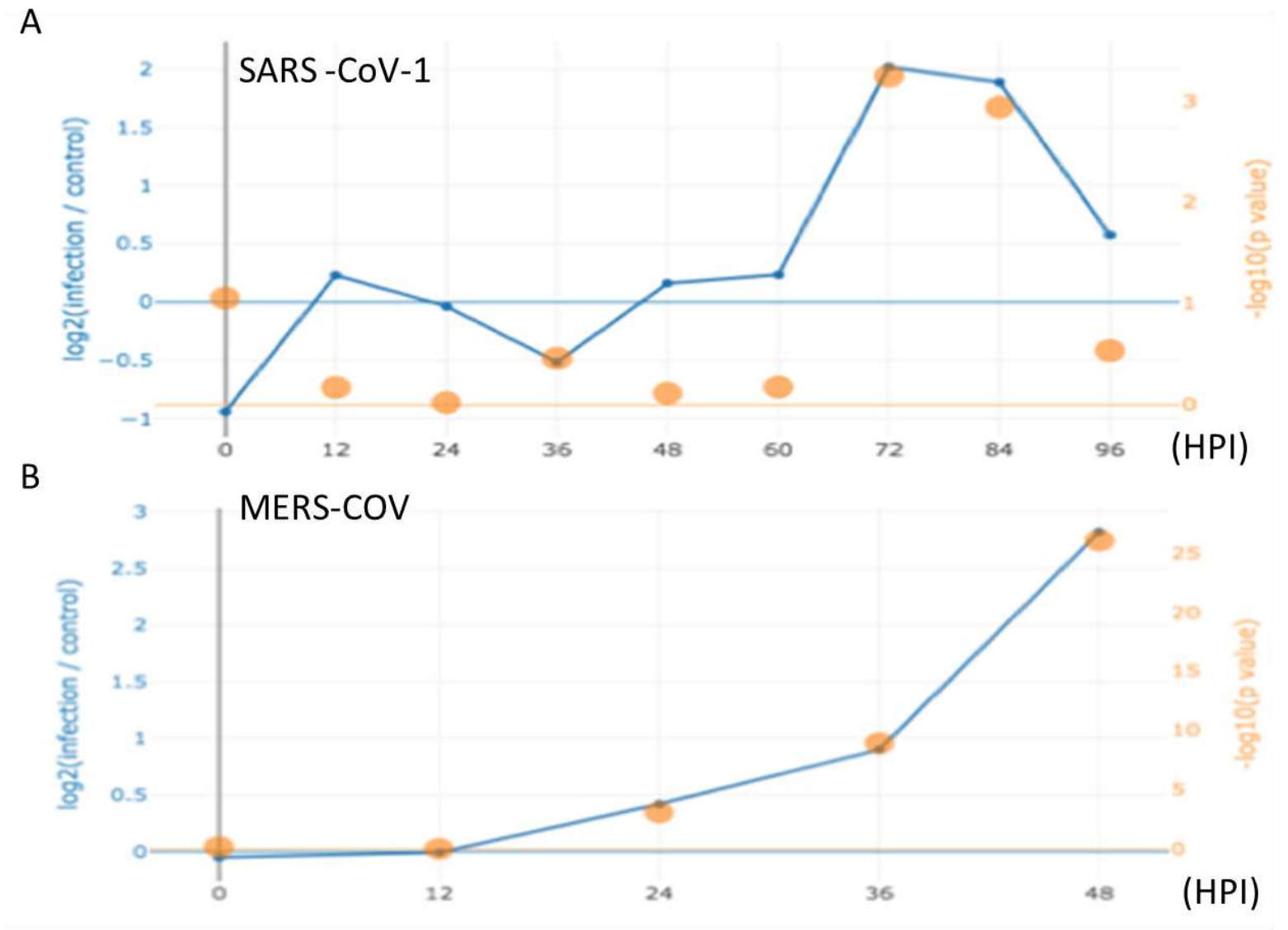

Figure 6 


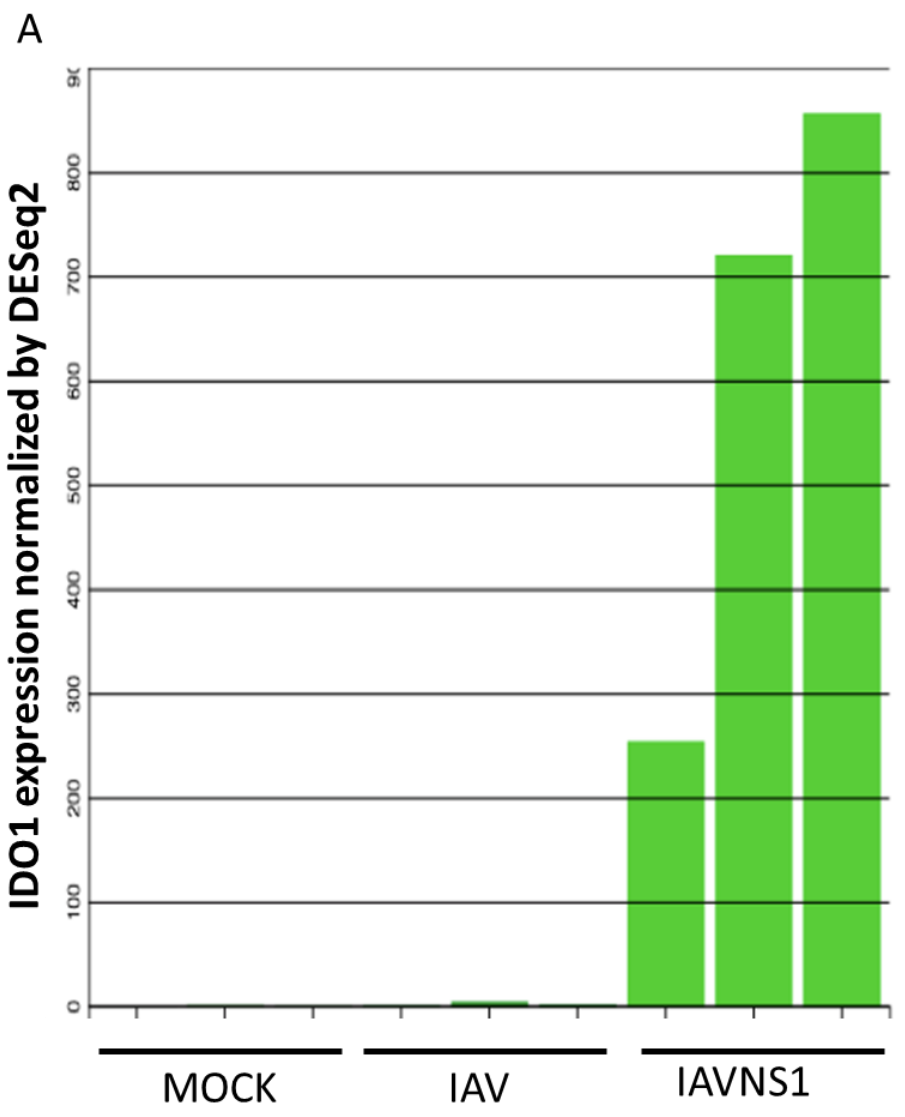

B


Figure 7 
A



B

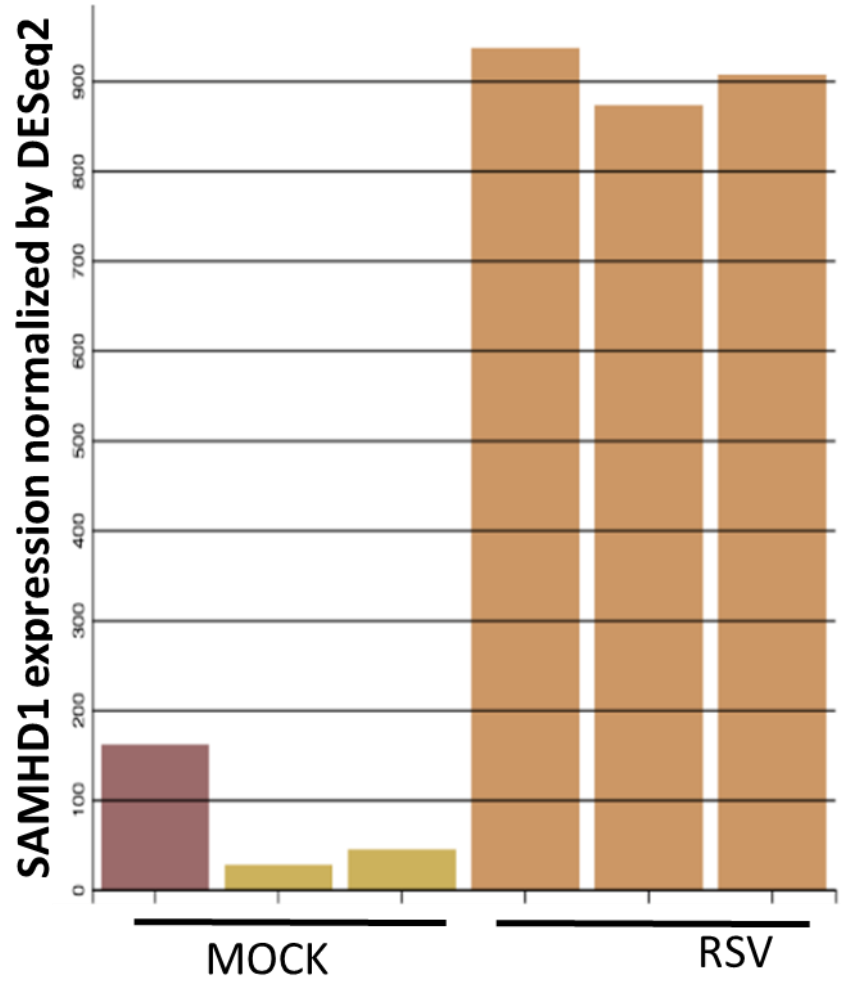

Figure 8 


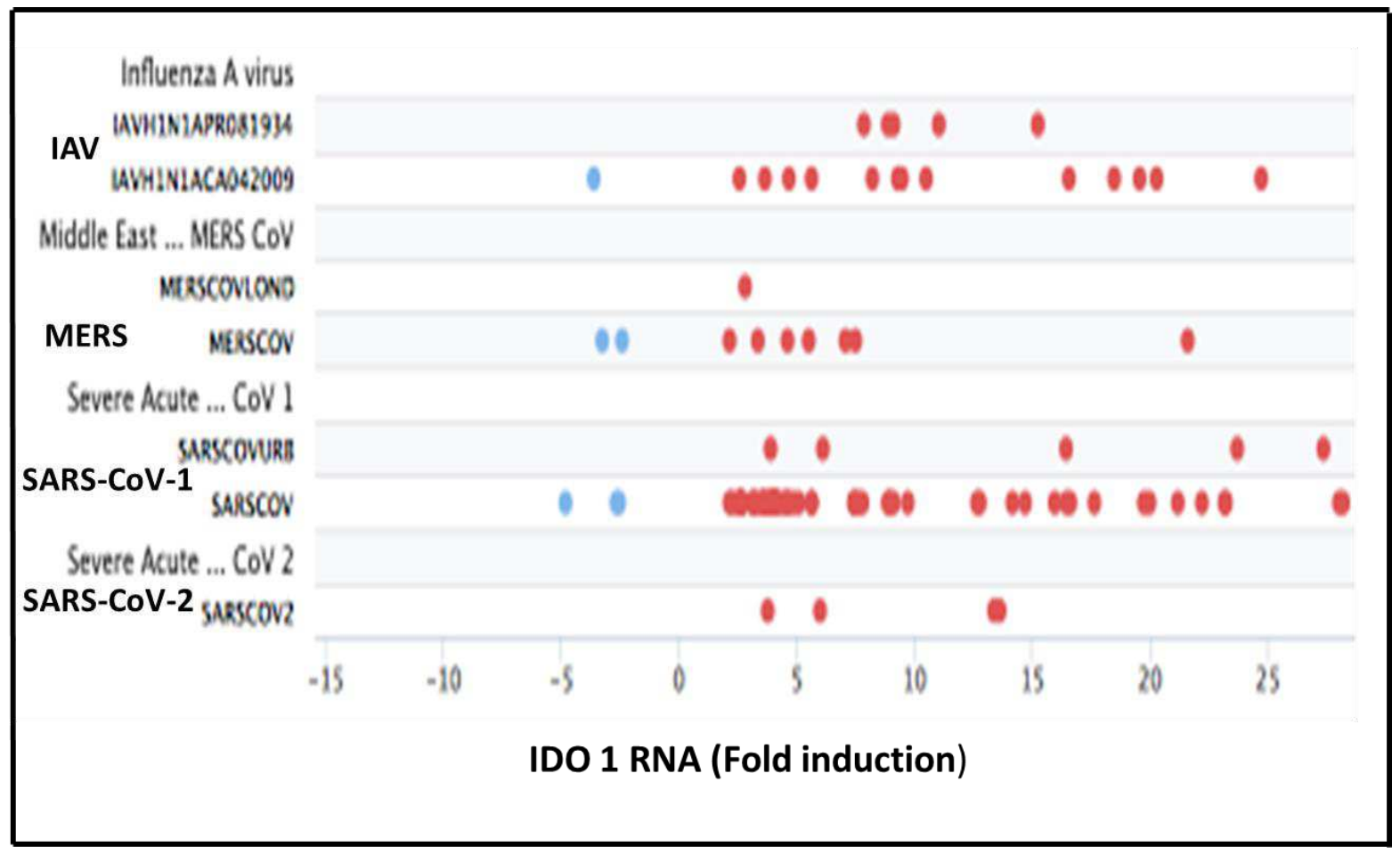

Figure 9 
SAMHD1

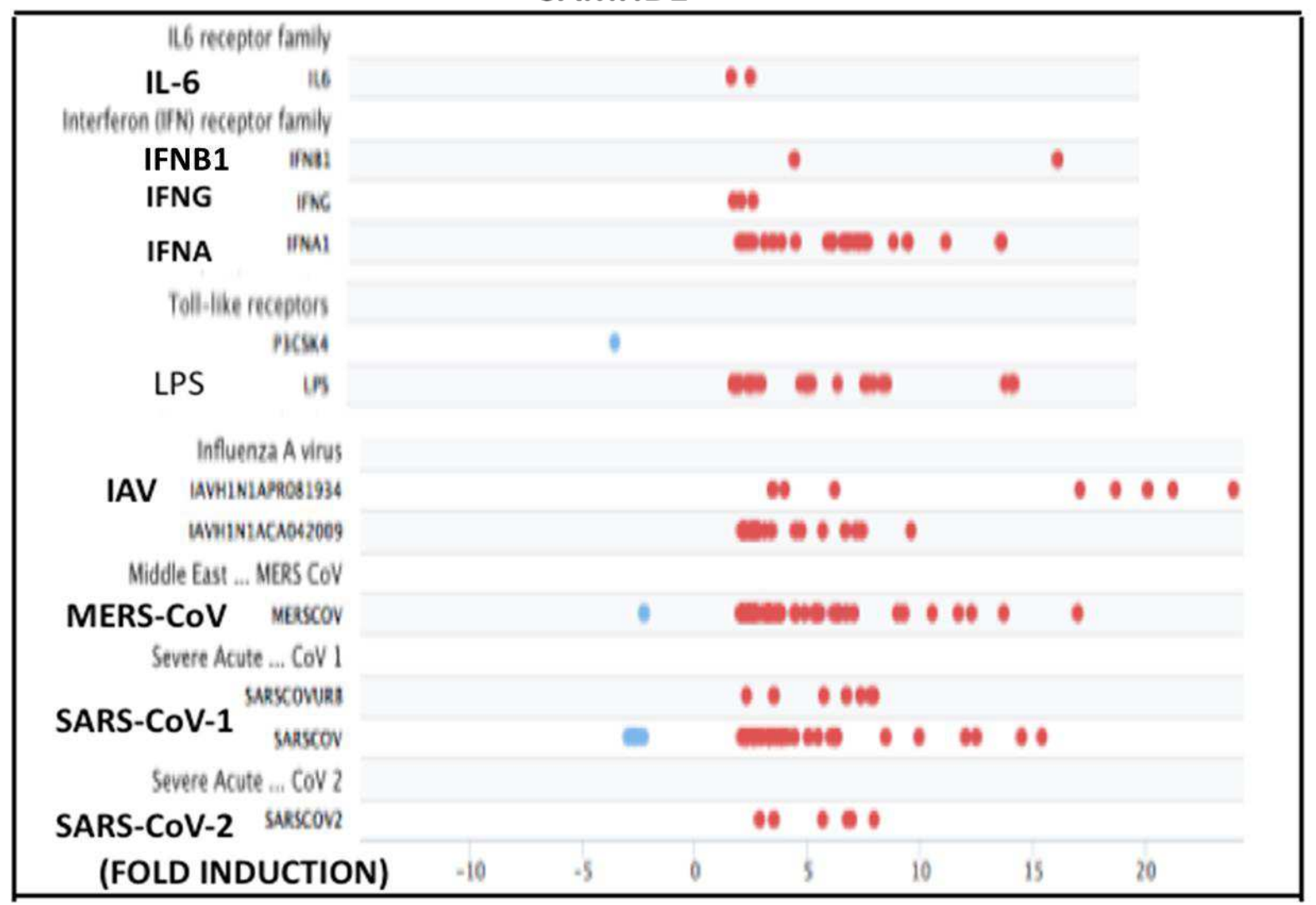

Figure 10 


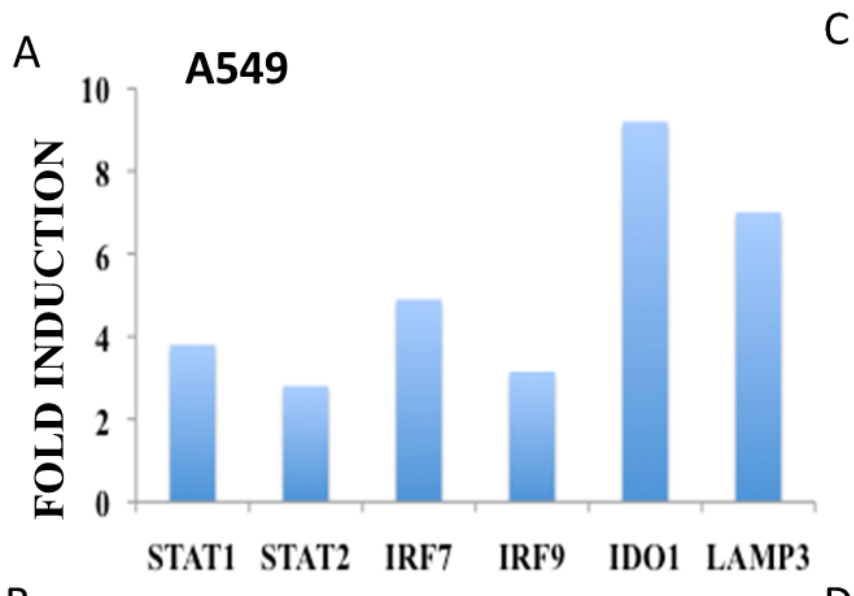

C
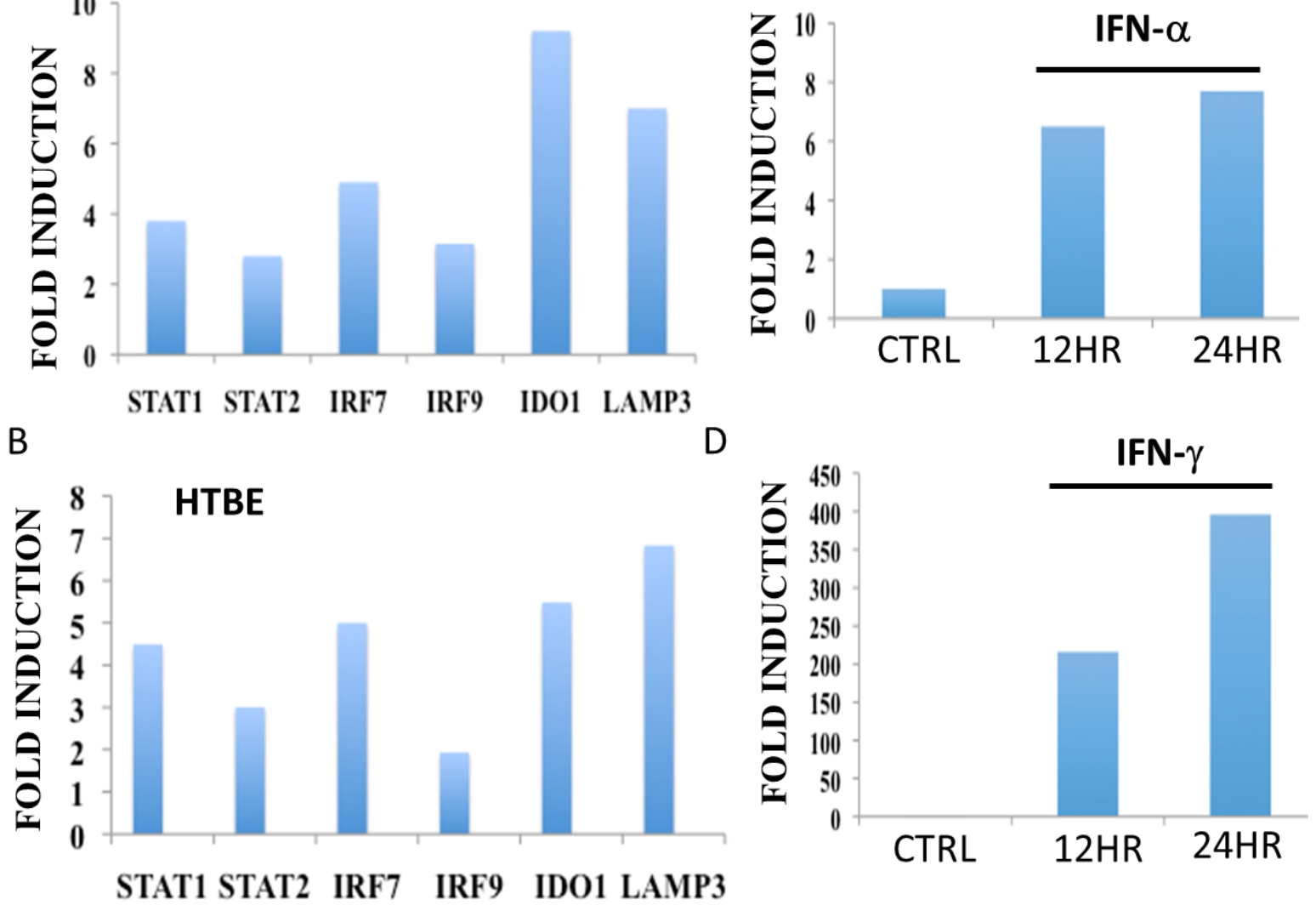

Figure 11 
A

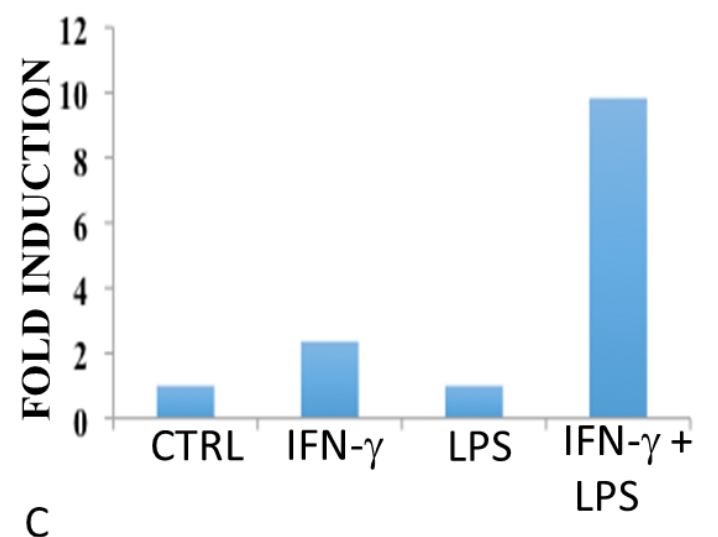

B

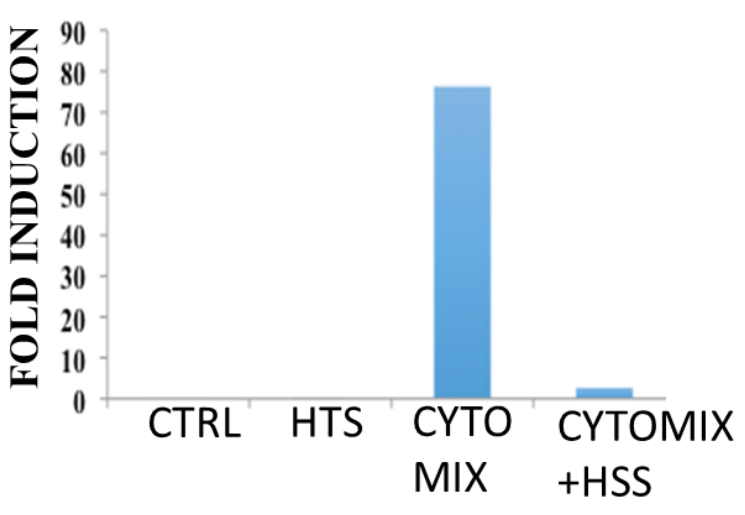

Human IDO1 gene promoter region

GAS C/EBP $\beta$

ISRE/GAS

I

$-1300$
ISRE C/EBP $\beta$

TSS

Figure 12 


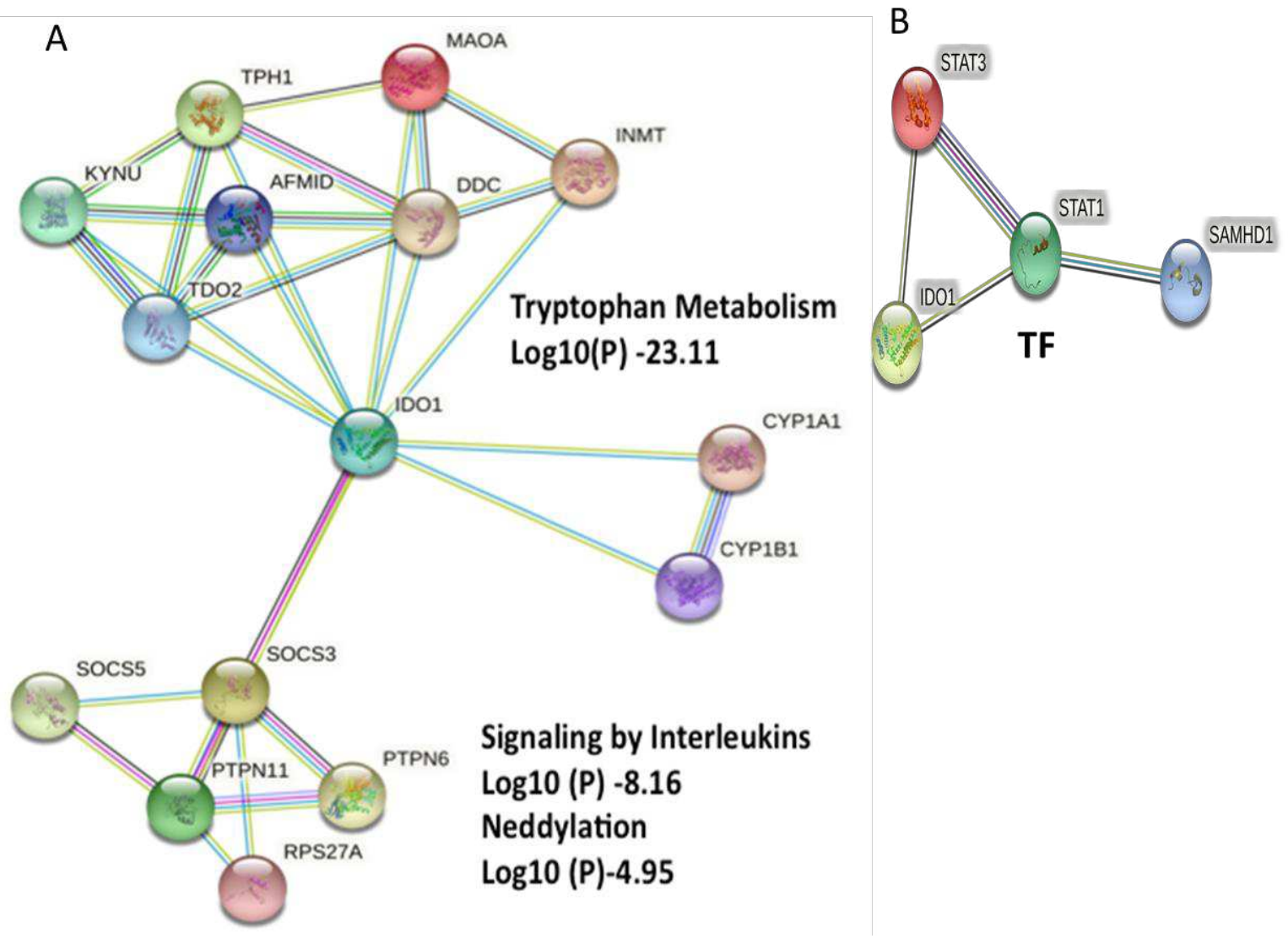

Figure 13 


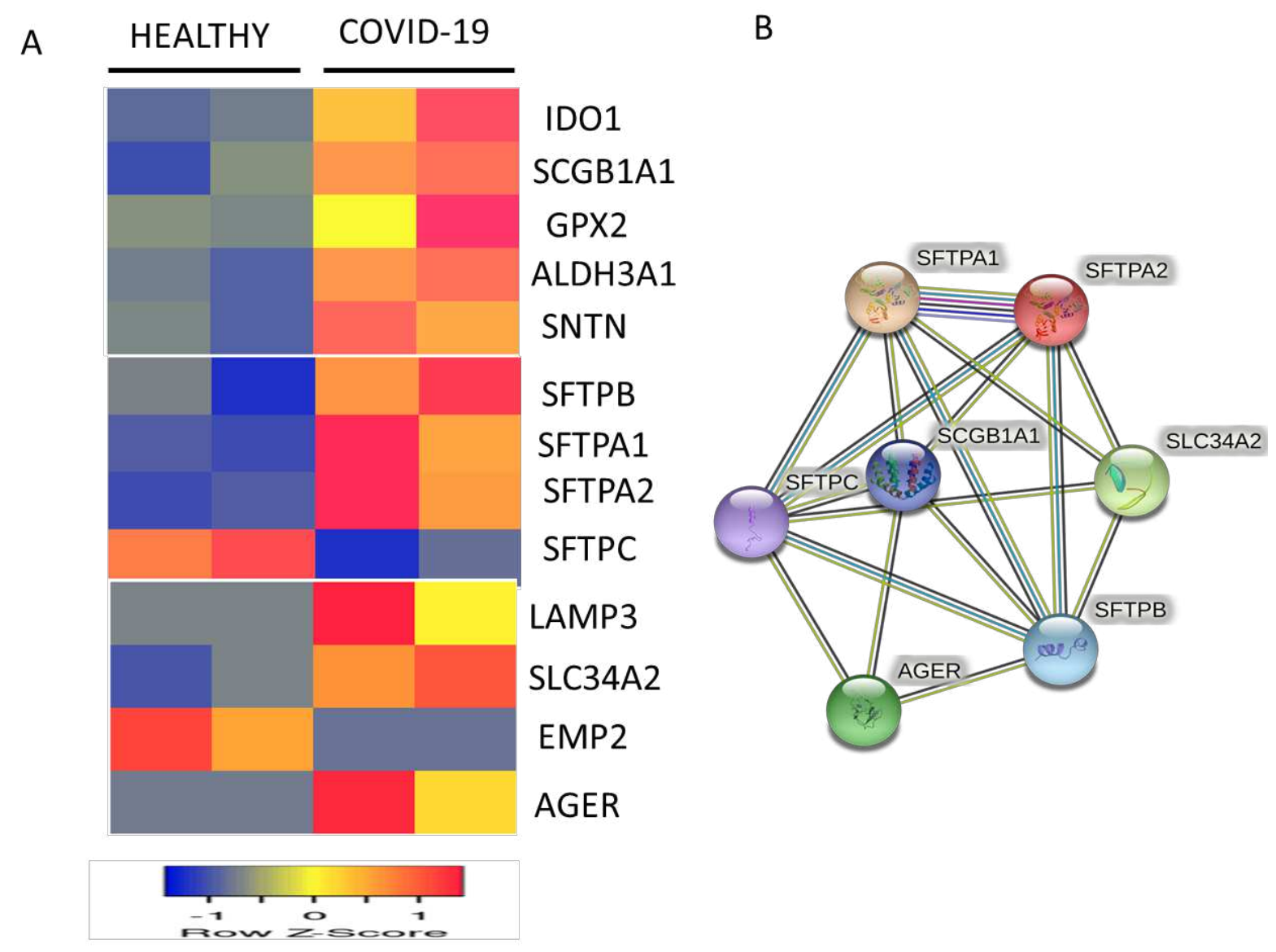

Figure 14 


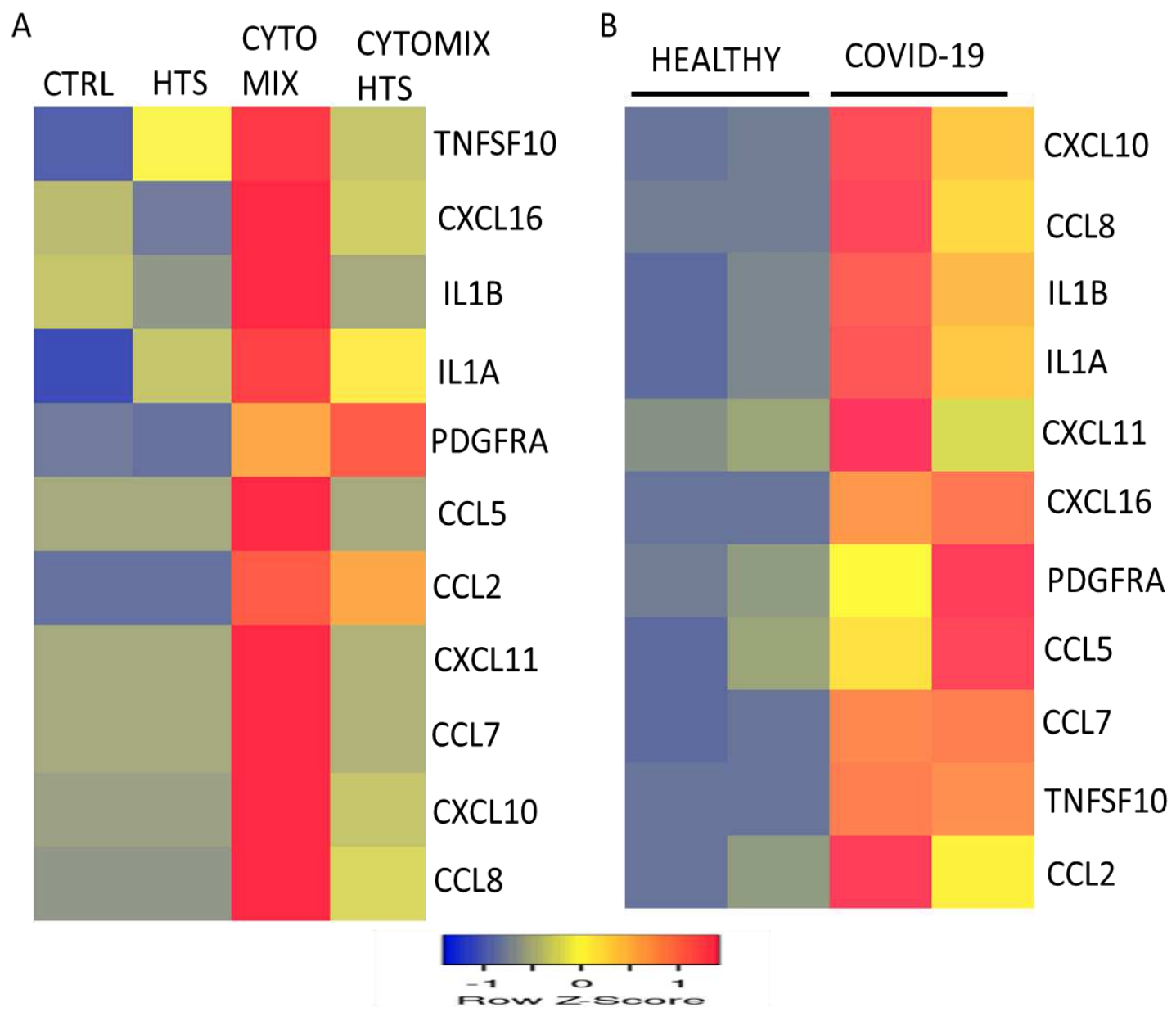

Figure 15 
A

B
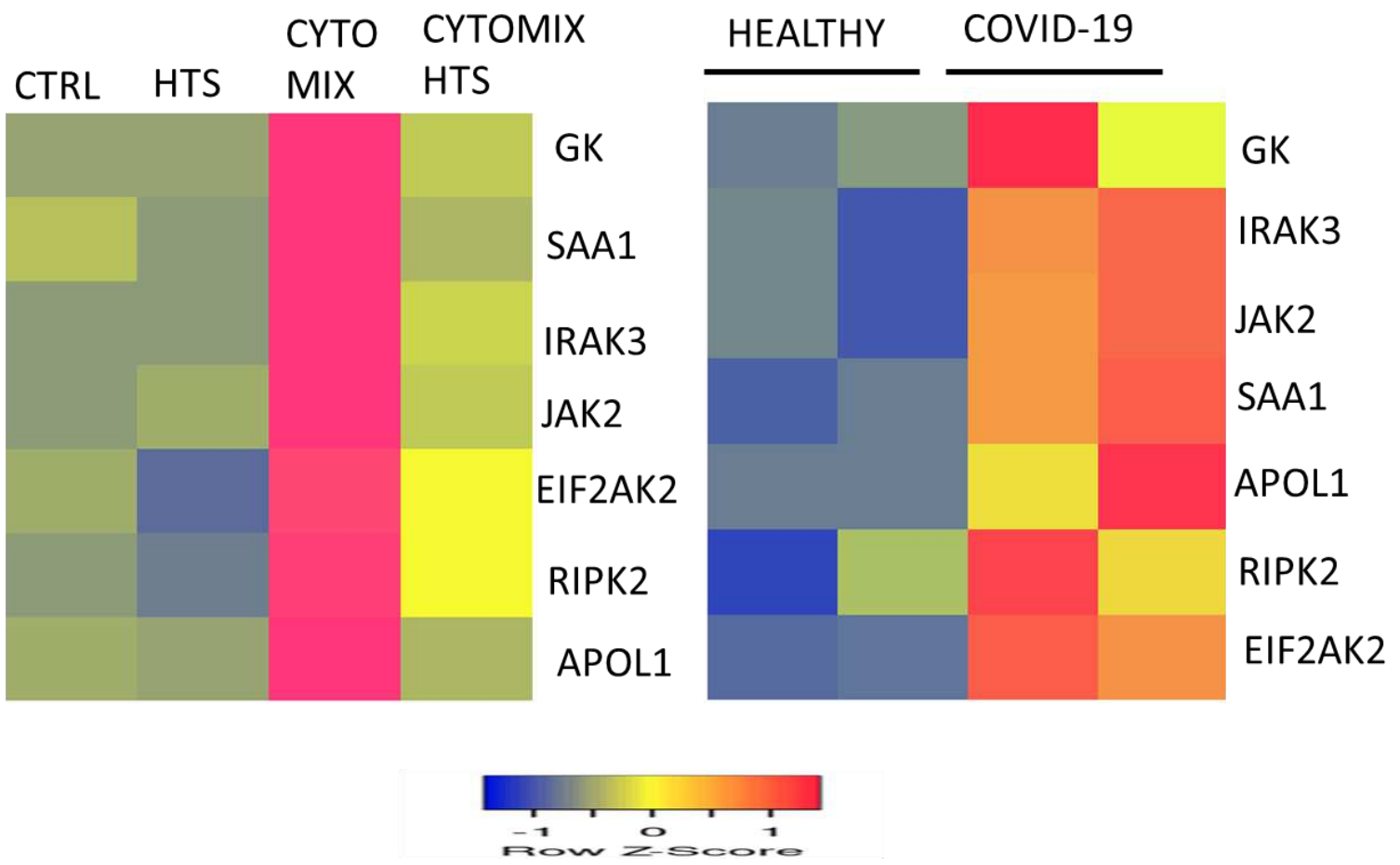

Figure 16 


\section{Supplementary Files}

This is a list of supplementary files associated with this preprint. Click to download.

- IDOsupplement.pdf 